\title{
Ordonomik als Methode zur Generierung von Überbietungsargumenten - Eine Illustration anhand der Flüchtlings(politik)debatte
}

Die Ordonomik versteht sich als Methode zur systematischen Generierung von Überbietungsargumenten. Solche Argumente können - als orthogonale Positionierung zu wertstrittigen Trade-off-Vorstellungen - einem Diskursversagen entgegenwirken. Dies ist wichtig im Hinblick auf Fragen der gesellschaftlichen (Selbst-)Steuerung durch eine (Re-)Formierung institutioneller Handlungsanreize sowie im Hinblick auf Fragen der gesellschaftlichen (Selbst-)Aufklärung durch eine (Re-)Formierung begrifflich sedimentierter Denkstrukturen.

Schlüsselbegriffe: Ordonomik, Diskursversagen, Überbietungsargumente, Ziel-Mittel-Konfusion, orthogonale Positionierung, Moralkommunikation

\section{Ordonomics as a Method for Generating Surpassing Arguments - An Illustration}

Ordonomics is a method for the systematic generation of arguments that are capable of surpassing normative value judgements. Surpassing arguments can cure discourse failures in the form of an orthogonal position to the mind-set of thinking in contested trade-offs. That is important for processes of societal (self-)governance via institutional reforms as well as for processes of societal (self-)enlightenment via reforms of idea(l)s.

Keywords: Ordonomics, Discourse Failure, Surpassing Arguments, Means-End Confusion, Orthogonal Position, Moral Communication

\section{Einleitung}

»Es gibt nicht nur falsche Antworten. Es gibt auch falsche Fragen.« Albrecht Fabri (2000: 121)

[1] Beginnen wir mit einem Gedankenexperiment: Nehmen wir an, wir empfangen Besuch von einem anderen Planeten. Unser Gast stellt sich vor als extraterrestrischer Kulturanthropologe mit einem Spezialinteresse für die Sitten und Gebräuche in Deutschland. Wir fühlen uns geschmeichelt und laden unseren Gast zu einem Fußballspiel der Bundesliga ein. Wir wissen nicht, dass er sich einer überirdischen Gleichheits-Heuristik verpflichtet fühlt. Aber wir merken schon bald, dass

\footnotetext{
Prof. Dr. Ingo Pies, Lehrstuhl für Wirtschaftsethik, Martin-Luther-Universität Halle-Wittenberg, Große Steinstraße 73, D-06099 Halle (Saale), Tel.: +49-(0)345-5523420, EMail: ingo.pies@wiwi.uni-halle.de, Forschungsschwerpunkte: Wirtschafts- und Unternehmensethik, Normative Institutionenökonomik, Ordonomik. - Für wertvolle kritische Hinweise herzlich zu danken ist Gerhard Engel, Sebastian Everding, Stefan Hielscher, Karl Homann, Ulrich Koester, Gerhard Minnameier, Nils Ole Oermann, Martina Pies, Christian Rennert, Daniel Streit, Vladislav Valentinov und Matthias Georg Will.
} 
unser Besucher blind zu sein scheint für die Schönheit des Fußballspiels, das sich als athletisches Drama vor unseren Augen entfaltet, in dem - und nota bene: indem - zwei talentierte und hoch motivierte Mannschaften aufeinandertreffen. Er ist auch blind für die Freude am Wettkampf, die die Zuschauer im Stadion empfinden. Sein professioneller Blick gilt allein den sozialen Beziehungen innerhalb einer Mannschaft. Und hier zeigt sich unser Gast schockiert von mehreren Befunden radikaler Ungleichheit, die ihm sofort ins Auge springen:

- Die Feldspieler laufen sich die Lunge aus dem Leib. Der Torwart hingegen steht zumeist rum.

- Der Torwart ist Chef im Strafraum und kommandiert die Abwehr.

- Der Torwart ist privilegiert. Er hat eine eigene Schutzzone (Fünf-MeterRaum).

- Der Torwart darf den Ball in die Hand nehmen und dafür sogar Handschuhe tragen.

[2] Gestützt auf diese Befunde lehnt unser empörter Gast die Institution des Fußballspiels als zutiefst ungerecht ab und hat sofort ein paar Gestaltungsvorschläge parat, wie man den diversen Tatbeständen eklatanter Ungleichbehandlung Abhilfe verschaffen könne, indem man die offenkundig ungehörige Privilegierung des Torwarts - und auch das damit verbundene Hierarchiegefälle innerhalb der Mannschaft - abschwächt oder gar auf null reduziert. ${ }^{1}$

[3] Wer im deutschen Sprach- und Kulturraum sozialisiert worden ist, hat ein intuitives Vorverständnis davon, was bei dieser extraterrestrischen Moralkommunikation schiefgelaufen ist. Denn hier gehört Fußball konstitutiv zur dritten Natur des Menschen - zur institutionellen Umwelt neben Instinkten und Emotionen als erster Natur und eingeübten Handlungsgewohnheiten als zweiter Natur. Folglich ist allen Lesern sogleich klar, dass die inkriminierten Ungleichheiten nicht als Ungerechtigkeiten einzustufen sind, weil sie sich im Fußballspiel - d.h. innerhalb eines institutionalisierten Systemkontexts, der uns zur dritten Natur geworden ist - als funktional erweisen. Zudem wird in Deutschland niemandem verwehrt, Torwart zu werden. Empirisch entscheiden sich allerdings die meisten Fußballer dagegen und suchen ihr Glück lieber als Feldspieler. Wir haben es hier also nicht mit einer unfairen Privilegierung zu tun, sondern mit einem Phänomen freiwilliger Selbst-Selektion. Insofern steht zu erwarten, dass die jenseitigen Ideal- und Reform-Vorstellungen unseres extraterrestrischen Besuchers nicht zu Besserungen des Fußballspiel(system)s führen würden, sondern allenfalls zu Verschlimmbesserungen. Sie verwirklichen zu wollen wäre nicht konstruktiv, sondern destruktiv und hätte zur Folge, den sozialen Sinn einer zum Nutzen aller erfolgreich einge-

1 Auf den ersten Blick liest es sich vielleicht wie eine Persiflage auf mein Gedankenexperiment - oder alternativ: wie ein Exempel für den Denkansatz, den mein Gedankenexperiment persifliert -, wenn Bernward Gesang (2017) einen jüngst veröffentlichten Aufsatz mit der normativen Setzung beginnt: »Unsere Art und Weise zu wirtschaften ist zutiefst ungerecht." Deshalb möchte ich betonen, dass ich das hier erstmals veröffentlichte Gedankenexperiment schon seit vielen Jahren in meinen Vorlesungen verwende - mit exakt diesem Wortlaut. 
spielten Praxis zu (zer-)stören, anstatt ihn schrittweise weiter zu kultivieren und so zur noch besseren Entfaltung zu bringen.

[4] Gerade aufgrund des vertrauten Systemkontexts ist dieses Gedankenexperiment geeignet, per Analogieschluss die Schwierigkeiten offenzulegen, die auftreten können, wenn man unvermittelt - ohne Systemverständnis - moralische Urteile und darauf aufbauende Empfehlungen zur marktwirtschaftlichen Praxis (von und innerhalb von Unternehmen) zu formulieren versucht, allein gestützt auf Intuitionen und die zugrunde liegenden Emotionen, die uns diverse Einzelheiten dieser Praxis leicht als ungehörig empfinden lassen. Der Hintergrund: Unsere Emotionen sind evolutionär entstanden. Sie sind darauf geeicht, uns in kleinen Gruppen überleben zu lassen. Im Vergleich zu dieser Entstehungsgeschichte haben wir es in der Marktwirtschaft - und allgemein: in der modernen (Welt-)Gesellschaft - mit hochartifiziellen Interaktionsumwelten zu tun, die sehr viel komplexer sind als Fußballspiele. Deshalb passieren uns hier sehr viel leichter solche Denkfehler, wie sie dem extraterrestrischen Besucher unterlaufen sind. Wir stoßen uns an Oberflächenphänomenen und übersehen dabei leicht den sozialen Sinn der Systeme. Gerade im Hinblick auf moralische Anliegen und deren Verwirklichung in der konkreten Alltagswelt gelingt es uns oft nicht, die srichtigen< Fragen zu stellen. Daran zeigt sich: Wir sind mit wichtigen Bereichen unserer dritten Natur (noch) nicht gut vertraut.

[5] Was beim Fußballspiel allen klar ist (insbesondere, weil hier weitestgehend Harmonie herrscht zwischen erster und dritter Natur), erscheint im Hinblick auf andere Funktionssysteme der modernen Gesellschaft - wie Wirtschaft, Politik, Wissenschaft usw. - vielen Menschen weitaus weniger selbstverständlich: Wettbewerb führt zur Emergenz genuin sozialer Phänomene, denn er bewirkt einen Hiatus zwischen den Handlungsmotiven einzelner Akteure (= Personen oder Organisationen) und ihren Handlungsergebnissen. Deshalb lassen sich gute Wettbewerbsergebnisse nicht auf gute Handlungsmotive - und analog: schlechte Wettbewerbsergebnisse nicht auf schlechte Handlungsmotive - zurückführen. Wer so fragt, stellt $>$ falsche $<$ Fragen, die (gerade auch in moralischer Hinsicht, vor allem durch verfehlte Zuschreibungen von Verantwortung und Schuld) in die Irre führen. ${ }^{2}$ Falsche Fragen haben zur Folge, dass viele Menschen unter dem Entfremdungsphänomen leiden, Kollisionen zwischen erster und dritter Natur zu empfinden: Die moderne Gesellschaft funktioniert - gerade aufgrund ihrer wettbewerblich strukturierten Funktionssysteme - über weite Strecken nun einmal hochgradig kontra-intuitiv.

2 Der Gesichtspunkt, auf den ich hier aufmerksam machen will, - dass Emotionen uns in die Irre führen können - ist bereits sehr früh und sehr pointiert von Friedrich Nietzsche (1881: 87, H.i.O.) zum Ausdruck gebracht worden. Er formuliert im zweiten Buch seiner »Morgenröte « als Aphorismus Nr. 99: » Worin wir Alle unvernünftig sind. - Wir ziehen immer noch die Folgerungen von Urteilen, die wir für falsch halten, von Lehren, an die wir nicht mehr glauben, - durch unsere Gefühle. « Zur Unterstützung dieser Sichtweise durch die neuere empirische Moralforschung vgl. grundlegend Haidt (2012) sowie Greene (2013). 
[6] Was heißt das für die Ethik, also für die Theorie der Moral? Wenn eine Individualethik wirtschaftlicher Handlungsmotive ungeeignet ist, wie sieht dann eine möglichst leistungsfähige Alternative aus? ${ }^{3}$ Welche Vorgehensweise bietet sich an, wenn man dem Wettbewerbselement in der modernen Wirtschaft - und allgemein: dem Wettbewerbselement in allen Funktionssystemen der modernen Gesellschaft, insbesondere in Politik, Öffentlichkeit, Zivilgesellschaft und Wissenschaft - systematisch Rechnung tragen will?

[7] Das ordonomische Forschungsprogramm gibt hierauf folgende Antwort: Die Ordonomik untersucht die Moraltauglichkeit der modernen Gesellschaft(sstruktur) sowie spiegelbildlich die Gesellschaftstauglichkeit der modernen Moral(kommunikation). Auf diesem Weg will sie zu zwei Lernprozessen beitragen, die sich wechselseitig ergänzen können: (a) zu institutionellen Reformen der Ordnung des Handelns sowie spiegelbildlich (b) zu begrifflichen Reformen der Ordnung des Denkens: Die Ordonomik versteht sich als Methode zur systematischen Generierung von Überbietungsargumenten (a) im Hinblick auf Fragen der gesellschaftlichen (Selbst-)Steuerung durch eine (Re-)Formierung institutioneller Handlungsanreize sowie (b) im Hinblick auf Fragen der gesellschaftlichen (Selbst-)Aufklärung durch eine (Re-)Formierung von Ideen und Begriffen sowie der ihnen zugrunde liegenden Denkkategorien.

[8] Das Forschungsprogramm der Ordonomik arbeitet mit einem Ensemble eng zusammenhängender (und sogar zusammengehöriger) Ideen. Es handelt sich um (a) eine Diagnose der Moderne, die die moderne Gesellschaft als innovative Wachstumsgesellschaft (und Unternehmen als Agenten innovativer Wertschöpfung im gesellschaftlichen Auftrag) kennzeichnet, (b) das Konzept >orthogonaler Positionierung`zur Überwindung umstrittener Werte-Trade-offs, (c) die Idee, den wettbewerbstypischen Hiatus zwischen Handlungsmotiv und Handlungsergebnis mit Modellen sozialer Dilemmata abzubilden, die die positive Analyse auf die nicht-intendierten Folgen intentionalen Handelns fokussieren, und schließlich (d) ein Drei-Ebenen-Modell, das die Möglichkeit sowohl gelingender als auch misslingender Lernprozesse in Rechnung stellt, namentlich in den Bereichen wirtschaftlicher Wertschöpfung (`Marktversagen`), kollektiv verbindlicher Regelsetzung (>Politikversagen $<$ ) und öffentlicher Meinungsbildung (>Diskursversagen $<$ ).

[9] Diesem ordonomischen Ansatz liegen Überlegungen zur Theoriebildungsstrategie zugrunde, die in einem umfangreichen Schrifttum ausgearbeitet und transparent gemacht worden sind. ${ }^{4}$ Dennoch finden sich in der kritischen Sekundärliteratur zahlreiche Missverständnisse (als jüngstes Beispiel vgl. Beschorner/ Kolmar 2016). Deshalb gehe ich nun anders als sonst vor, nämlich weniger

3 Zur ordonomischen Kennzeichnung eines komplementären Verhältnisses zwischen Individualethik und Institutionenethik vgl. Pies (2000b; 2013 und 2016b).

4 Ich füge hier nur einige neuere Buchpublikationen an. Vgl. Pies (2009a; 2009b; 2012a; 2015a). Für zahlreiche theoriestrategische Überlegungen, die die Ordonomik geprägt haben, vgl. Pies (2016a). Einen Überblick über Peer-Review-Artikel zur Ordonomik findet man unter http://ethik.wiwi.uni-halle.de/2166_199920/2166_199912/ (zuletzt abgerufen am 16.3.2017). 
deduktiv und stattdessen stärker induktiv: Ich beginne mit einigen empirischen Belegen für Diskursversagen (Abschnitt 2). Dann kennzeichne ich anhand einer sehr knapp gehaltenen Auseinandersetzung mit den Schriften von Thomas Hobbes drei ordonomische Theorie-Elemente - die Ziel-Mittel-Konfusion, das Überbietungsargument und den institutionellen Reformvorschlag (Abschnitt 3). Diese drei Elemente sollen mir dabei helfen, im nächsten Schritt anhand des konkreten Beispiels der Flüchtlings(politik)debatte illustrativ vor Augen zu führen, wie die ordonomische Vorgehensweise beschaffen ist, mit der versucht werden kann, Diskursblockaden methodisch aufzulösen (Abschnitt 4). Die dann noch folgenden Abschnitte widmen sich einer Reflexion (Abschnitt 5) und einem abschließenden Ausblick (Abschnitt 6).

\section{Finanzkrise, Eurokrise, Flüchtlingskrise - Belege für Diskursversagen}

[10] Die Bundesrepublik hat in den letzten zehn Jahren nicht weniger als drei internationale Krisen durchlebt: die große Finanzmarktkrise der Jahre 2007ff., die ab 2010 offenkundig gewordene Eurokrise und die schon seit vielen Jahren schwelende europäische Flüchtlingskrise, die (aus deutscher Sicht) im Jahr 2015 mit der zeitweisen Öffnung der Balkanroute vorübergehend eskaliert ist.

[11] Die erste dieser Krisen hat den Adrenalinpegel und die Tonlage des öffentlichen Diskurses nachhaltig verändert (vgl. Judis 2016) - und damit auch die Rahmenbedingungen, mit denen sich Versuche konfrontiert sehen, wirtschaftsethische Aufklärung zu betreiben. Im Zuge dieser - hinsichtlich ihrer Ursachen oft missverstandenen und daher viele Bürger ratlos, hilflos oder schlicht wütend machenden - Krise ist es zu einem dramatischen Ansehensverlust mehrerer Berufsgruppen gekommen. Banker haben seitdem eine schlechte Presse. Aber auch wirtschaftliche Führungskräfte generell haben einen Reputationsverlust erlitten. Das Bild vom sgierigen Manager ist im öffentlichen Diskurs zum feststehenden Stereotyp geworden (vgl. kritisch hierzu Pies 2015 [2010] sowie 2015b).

[12] Ähnlich wie schon nach der Weltwirtschaftskrise der Jahre 1929ff. ist durch die große Finanzmarktkrise nicht nur die Wirtschaft, sondern auch die Wirtschaftswissenschaft in Mitleidenschaft gezogen worden. Viele machen es der Ökonomik zum Vorwurf, diese Krise nicht vorausgesagt zu haben - ohne sich von dem ökonomischen Gedanken irritieren zu lassen, dass eine Finanzmarktkrise, die man sicher voraussagen kann, so nicht eintreten würde. In der Öffentlichkeit ist es zwischenzeitlich zu einem beliebten Sport geworden, sich über ökonomische Theoriebildung lustig zu machen - wobei allerdings nicht zu übersehen ist, dass sich die Theoriekenntnisse der Kritiker und die Lautstärke radikaler Kritik geradewegs umgekehrt proportional zueinander verhalten. Dabei gäbe es einigen Grund, vor der Ökonomik den Hut zu ziehen: Nach Ausbruch der Krise, markiert durch die Lehman-Pleite, kam es binnen weniger Tage zu weltweit abgestimmten Rettungsaktionen, ohne dass zuvor ein gemeinsames Drehbuch für das akute Krisenmanagement verabredet worden wäre. Man könnte dies mit Fug und Recht als einen Triumph ökonomischer Forschung und Lehre interpretieren: Ohne eine im 
globalen Kontext hinreichend einheitliche Vorstellung, wie sinnvolle Rettungsaktionen auszusehen haben, wäre dies nicht möglich gewesen. ${ }^{5}$

[13] Zu den zeitgenössischen Kuriositäten gehört, dass der Ökonomik nicht zugutegehalten wird, vor der zweiten Krise sehr eindringlich gewarnt zu haben. Dabei ist genau dies der Fall. Erinnert sei nur an den offenen Brief vom 9. Februar 1998, mit dem 155 Professoren der Wirtschaftswissenschaften an die Öffentlichkeit traten, um zu warnen: »Der Euro kommt zu früh « ${ }^{6}$. Zusätzlich verwiesen sei auf den hellsichtigen Aufsatz von Milton Friedman, der schon ein Jahr zuvor veröffentlicht worden war. In ihm warnt Friedman vor einer fatalen ZielMittel-Konfusion: Die europäische Währungsunion sei dem Anliegen einer politischen Friedensunion nicht zuträglich, sondern abträglich, weil sie innerhalb der Mitgliedsländer zwischenstaatliche Konflikte heraufbeschwöre. Aufgrund nicht leicht zu bewältigender Governanceprobleme werde sich die Währungsunion nicht als Kitt, sondern als Sprengstoff erweisen. ${ }^{7}$

[14] Die dritte Krise teilt mit der zweiten eine wichtige Gemeinsamkeit, wenn man auf die Frontstellung der öffentlichen Debatte schaut: In beiden Fällen gibt es eine radikale Aufteilung in zwei Lager - pro und contra Regierungspolitik. In beiden Fällen halten die extremen Kontrahenten jeweils sich selbst für moralisch gut, während sie ihren Gegenpart als unmoralisch einstufen: In beiden Fällen wirft die Contra-Seite der Regierung vor, die Interessen des deutschen Volkes zu verraten. Und in beiden Fällen wirft die Pro-Seite den Regierungskritikern vor, sich vom demokratischen Verfassungskonsens zu verabschieden. Abbildung 1 hilft, die beiden smental models zu kennzeichnen, die hier jeweils aufeinanderprallen. Die Pro-Seite ist so eingezeichnet, wie sie von der Contra-Seite wahrgenommen wird und umgekehrt.

[15] Im Hinblick auf die zweite Krise erheben Kritiker der Regierungspolitik den Vorwurf, dass die Art der Griechenland-Rettung das Versprechen bricht, in der europäischen Währungsunion gebe es kein sbail-out‘, d.h. keine Vergemeinschaftung von Staatsschulden. Sie vertreten die Interpretation, dass die Bundesregierung die Interessen der Griechen höher gewichtet als die Interessen der Deutschen. Viele Bürger fühlen sich auf unrechtmäßige Weise in Haftung genommen.

5 Freilich gibt es noch zahlreiche weitere Gründe, der Ökonomik als Wissenschaftsdisziplin eine Anerkennung zu zollen, die ihr gegenwärtig zumeist versagt bleibt. Für ihre gemeinwohlfördernden Wirkungen auf Wirtschaft und Gesellschaft vgl. z.B. Litan (2014) sowie - sehr kurz und anschaulich - den Blogbeitrag von o.A. (2017).

6 Vgl. Aurecon (1998).

7 Vgl. Friedman (1997). Sein Schlussabsatz ist auch heute noch bemerkenswert. Er lautet: »The drive for the Euro has been motivated by politics not economics. The aim has been to link Germany and France so closely as to make a future European war impossible, and to set the stage for a federal United States of Europe. I believe that adoption of the Euro would have the opposite effect. It would exacerbate political tensions by converting divergent shocks that could have been readily accommodated by exchange rate changes into divisive political issues. Political unity can pave the way for monetary unity. Monetary unity imposed under unfavorable conditions will prove a barrier to the achievement of political unity. « 
Graphisch interpretiert bedeutet dies, dass die Pro-Seite im Trade-off rechts unten verortet wird. Im Hinblick auf die dritte Krise wird diese Frontstellung dupliziert. Hier erheben Kritiker der Regierungspolitik den Vorwurf, dass die Art der Flüchtlingsrettung das Versprechen bricht, in Europa gebe es sichere Grenzen. Sie vertreten wiederum die Interpretation, dass die Bundesregierung die Interessen der Flüchtlinge höher gewichtet als die Interessen der Deutschen. Der Kanzlerin wird vorgeworfen, ihren Amtseid zu verletzen, Schaden vom deutschen Volk abzuwenden. Graphisch interpretiert bedeutet dies, dass die Pro-Seite im Trade-off erneut rechts unten verortet wird.

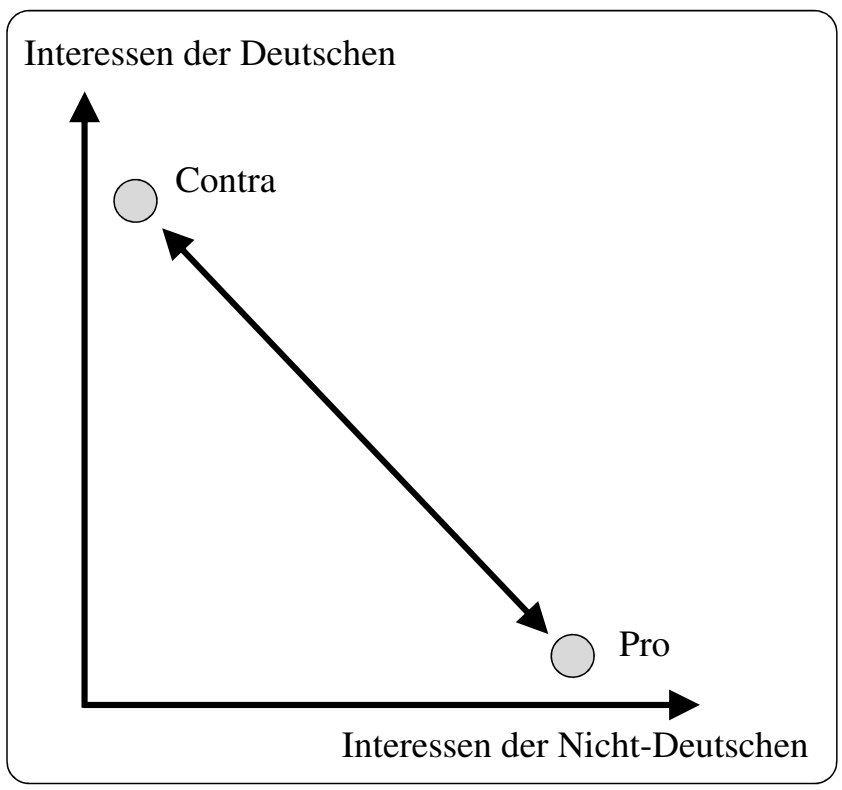

Abbildung 1: Die wertstrittige Frontstellung der öffentlichen Debatte (Quelle: eigene Darstellung)

[16] Blickt man auf die Kommunikation der jeweiligen Pro-Seite, so verhält es sich genau spiegelverkehrt. Im Hinblick auf die zweite Krise machen die Befürworter der Regierungspolitik ihren Kritikern den Vorwurf, deutsche Interessen über griechische Interessen zu stellen und sich mit diesem engherzigen (und engstirnigen) Nationalismus vom europäischen Anliegen einer politischen Friedensunion verabschiedet zu haben. Dieser Interpretation zufolge ist die Contra-Seite im Trade-off links oben zu verorten. Auch diese Sichtweise wird dupliziert, wenn man sich der dritten Krise zuwendet. Hier wird die Kritik an der Regierungspolitik zur Rettung von Flüchtlingen aufgefasst als Ausdruck anti-humanitärer Fremdenfeindlichkeit, als >rassistisch Solidarität und als Abkehr von der Idee universaler Menschenrechte. Graphisch 
interpretiert bedeutet dies, dass die Contra-Seite im Trade-off erneut links oben verortet wird.

[17] Das Ergebnis dieser diskursiven Frontstellung sind zunehmend tiefe Zerwürfnisse zwischen den beiden entzweiten Lagern. Die politische Debatte ist immer weniger ein gemeinsames Ringen der Demokraten um das bessere Argument. Stattdessen greift Bunkermentalität um sich: Die Positionen werden verfestigt. Man gräbt sich ein und zementiert die eigene Argumentationslinie. Wer sich selbst für 'gut< hält und die andere Seite für unmoralisch, hört nicht mehr zu und spricht lieber über die andere Seite als mit ihr. Diese Form der Auseinandersetzung verbindet nicht. Sie spaltet. Der Diskurs versagt.

[18] Eine interessante (und beunruhigende) Begleiterscheinung dieses Diskursversagens besteht darin, dass viele Menschen auf der Contra-Seite Zeitungen und Fernsehen nicht (mehr) als neutrale >Medien wahrnehmen, sondern als parteiische Instanzen. Ihr Motto lautet: `Lügenpresse ‘. Sie sehen sich mit ihren (moralischen) Anliegen in der veröffentlichten Meinung nicht mehr angemessen repräsentiert. Sie fühlen sich verunglimpft. Und umgekehrt empfinden viele Menschen auf der Pro-Seite manche Kommunikationsformen im Bereich der 'Social Mediar, die oft die Funktion einer Art inoffizieller >Gegen-Öffentlichkeit` übernehmen, als Ausdrucksform von 'Wutbürgern`, Verschwörungstheorien, 'hate speech` und 'fake news`. Auch hier schreitet die Entzweiung weiter voran.

[19] Im Hinblick auf den aktuellen Rand dieser zeitgenössischen Phänomene formuliert die Ordonomik folgende Diagnose: Die schnelle Aufeinanderfolge von Finanzkrise, Eurokrise und Flüchtlingskrise hat den öffentlichen Meinungsbildungsprozess in Deutschland partiell entgleisen lassen. Hier liegt Diskursversagen vor. Dieses Diskursversagen ist das Resultat davon, dass beide Seiten - Pro und Contra - seit geraumer Zeit auf eine forcierte Moralisierung politischer Themen gesetzt haben, wodurch man sich selbst ins Recht und den politischen Gegner ins Unrecht zu setzen versuchte, so als ließen sich nicht auch für dessen Anliegen moralische Überlegungen und gute Gründe ins Feld führen.

[20] Ordonomisch betrachtet besteht ein Grundübel der Debatte darin, dass beide Seiten sich so verhalten, als hätten sie schon längst den Versuch aufgegeben, sich wechselseitig überzeugen zu wollen. Die jeweiligen Argumentationsstrategien sind jedenfalls ganz anders ausgerichtet. Man betreibt vornehmlich Binnen-Kommunikation: In beiden Lagern wird mit Bekundungsargumenten und Werturteilen gearbeitet, die darauf setzen, die jeweils eigene Position moralisch aufzuwerten und die gegnerische Position moralisch abzuwerten. Die gegnerische Auffassung wird nicht inhaltlich erörtert (und sachlich zu widerlegen versucht). Stattdessen wird sie tabuisiert - und damit vom Diskurs ausgeschlossen. Auf beiden Seiten setzen viele Akteure nicht auf Argumentation, sondern auf Argumentationsverweigerung. Man bemüht die Kategorien von Gut und Böse, denkt in FreundFeind-Schemata und betreibt in moralischer Selbstgerechtigkeit mit offenkundig reinem Gewissen ein normatives >upgrading der eigenen und ein normatives >downgrading $<$ der anderen Seite.

[21] Da beide Seiten innerhalb des Trade-off, der als Denkrahmen gar nicht in Frage gestellt wird, mit strukturanalogen Kommunikationsstrategien arbeiten, 
kommt es zu einem Diskursversagen, das die Form annimmt, den politischen Streit eskalieren zu lassen. Anstatt auf die Annäherung an einen demokratischen Konsens wird die politische Auseinandersetzung so auf Dissens programmiert. Die politischen Gräben werden nicht überbrückt, sondern tiefer ausgehoben. Kurz: Es fehlt an >orthogonaler Positionierung‘. Im Diskurs herrscht - auf beiden Seiten - ein Mangel an Überbietungsargumenten, die zumindest potenziell die Kraft aufweisen, alle Teilnehmer am Diskurs überzeugen zu wollen und überzeugen zu können.

Um nun zu zeigen, dass - und vor allem: wie - die Ordonomik zur Lösung dieses Problems beizutragen vermag, geben die folgenden zwei Abschnitte eine Antwort auf folgende Fragen:

- Abschnitt 3: Welche besondere diskursive Qualität hat ein Überbietungsargument? Wie lassen sich moralische Ziel-Mittel-Konfusionen überwinden? Inwiefern schaffen institutionelle Reformen die Voraussetzung zur Verwirklichung normativ berechtigter Anliegen?

- Abschnitt 4: Wie kann man durch Einsatz der ordonomischen Methode eine orthogonale Positionierung zur diskursiven Frontlinie der Flüchtlings(politik)debatte erarbeiten?

\section{Thomas Hobbes und die Überwindung von Ziel-Mittel-Konfusionen in der Friedenspolitik mittels Überbietungsargument und institutioneller Reform ${ }^{8}$}

[22] An dieser Stelle sei ein kurzer Hinweis erlaubt: Im Folgenden geht es mir um eine problemorientierte Einführung in drei Theorie-Elemente der spezifisch ordonomischen Denkweise. Ich hoffe, dass diese Denkweise sich leichter erschließen lässt, wenn ich ausweise, inwiefern die Ordonomik sich einzelner TheorieElemente bedient, die man - gemäß (m)einer ganz bestimmten, höchst unkonventionellen Lesart - schon bei Thomas Hobbes auffinden kann. Es geht mir in diesem Abschnitt also nicht um Ideengeschichte, sondern um eine möglichst anschauliche Erläuterung, für exakt welche Problemstellungen die drei TheorieElemente als Lösungskonzepte bereits von Thomas Hobbes zum Einsatz gebracht wurden.

[23] Thomas Hobbes gehört zu den am schlechtesten beleumundeten Autoren der Philosophiegeschichte. Ihm wird vorgeworfen, was man seit geraumer Zeit auch gerne der Ökonomik vorwirft: mit einem einseitigen - und zudem radikal verfehlten - Menschenbild zu arbeiten. Besonders übel genommen hat man ihm die Aussage, dass der Mensch dem Menschen ein Wolf sei (`Homo homini Lupus $<$. Diese Aussage findet sich im Widmungsschreiben seines Buches $>$ De Cive «. Dabei wird oft übersehen, dass nicht nur im gleichen Widmungsschreiben, sondern sogar im gleichen Satz (!) dieses Schreibens noch eine weitere Aussage getroffen wird. Sie lautet, dass der Mensch dem Menschen ein Gott sei (’Homo

8 Zur Argumentation dieses Abschnitts vgl. ausführlich Pies (2012c) sowie Homann (2014: insbes. 117-132 und 141-150). 
homini Deus $)$. In der deutschen Übersetzung liest sich die Passage wie folgt (Hobbes 2014 [1642-1658]: 62f., H.i.O.]):

»Nun sind sicher beide Sätze wahr: Der Mensch ist ein Gott für den Menschen, und: Der Mensch ist ein Wolf für den Menschen; jener, wenn man die Bürger untereinander, dieser, wenn man die Staaten untereinander vergleicht. Dort nähert man sich durch Gerechtigkeit, Liebe und alle Tugenden des Friedens der Ähnlichkeit mit Gott; hier müssen selbst die Guten bei der Verdorbenheit der Schlechten ihres Schutzes wegen die kriegerischen Tugenden, die Gewalt und die List, d.h. die Raubsucht der wilden Tiere, zu Hilfe nehmen. Wenn auch die Menschen sich dies gegenseitig zum Vorwurf machen, weil sie nach einem eingeborenen Hang die eignen Handlungen, von andern verübt, wie in einem Spiegel anschauen, wo das Linke rechts und das Rechte links erscheint, so ist es doch nach dem in der Notwendigkeit der Selbsterhaltung wurzelnden Naturrecht nicht als Schuld anzusehen."

[24] Auf den ersten Blick könnte es wie ein Paradoxon anmuten, dass Hobbes in der Tat beide Aussagen gleichzeitig vertritt. Der vermeintliche Widerspruch lässt sich jedoch leicht auflösen: Hobbes war Situationstheoretiker. In der Situation eines verfassten Gemeinwesens gilt für ihn `Homo homini Deus‘. Hier tendieren die Menschen zum Frieden. Sie begegnen einander mit Gerechtigkeit und Liebe. Und in der Situation einer Anarchie gilt für ihn >Homo homini Lupusく. Hier tendieren die Menschen zum Krieg. Sie begegnen einander mit List und Gewalt.

[25] Es ist also offenkundig Unsinn, wenn Hobbes ein negatives Menschenbild oder eine pessimistische Psychologie unterstellt wird. Seine einschlägigen Aussagen hierzu sind - ganz in Analogie zum homo oeconomicus der Ökonomik ${ }^{9}$ nicht als Anthropologie zu lesen, nicht als Aussage über die Natur oder das Wesen des Menschen, sondern als Aussage über Situationseigenschaften. Folglich empfiehlt es sich, Hobbes strikt im Hinblick auf die von ihm ausgewiesene Fragestellung zu interpretieren. Und diese Fragestellung gilt ganz explizit dem Erkenntnisinteresse, wie sich die Situation des (Bürger-)Krieges in eine Situation des Friedens überführen lässt. Gemünzt auf die englischen Verhältnisse zur Mitte des 17. Jahrhunderts, und formuliert in der für die damalige Zeit typischen christlichen Diktion (und zudem unter Bedingungen der Zensur!), geht es ihm um die Frage: Wie kann man Bürger in die Lage versetzen, sich nicht länger als Wölfe zu begegnen, sondern als Ebenbilder Gottes? Säkular ausgedrückt: Wie kann man bewirken, dass der Mensch dem Menschen ein Mensch sei?

[26] Zur Beantwortung dieser Frage entwickelt Hobbes eine Theorie der Situation - genauer: eine Theorie der Situationstransformation. Diese Theorie lässt sich am besten verstehen, wenn man sie auf das Problem bezieht, wie sich eine moralische Ziel-Mittel-Konfusion überwinden lässt. Hierbei hilft Abbildung 2. Sie bildet die dilemmatische Problemsituation zweier Parteien ab, die sich im (Bürger-)Krieg miteinander befinden und vor der Frage stehen, ob sie die Waffen ruhen lassen

9 Vgl. hierzu Becker (1982 [1976]), Stigler/Becker (1996 [1977]), Zintl (1989), Pies (1993: 86-197), (2015 [2006]) sowie Homann (2002 [1994]). Vgl. auch McKenzie (2010) sowie Pies/Hielscher (2014). Zum Rational-Choice-Modell und seiner interdisziplinären Bedeutung für die gesamten Sozialwissenschaften vgl. Bowles (2004) sowie insbesondere Gintis $(2009 ; 2017 a ; 2017 b)$. 
sollen. Um ein soziales Dilemma handelt es sich, weil das Gleichgewicht in diesem Spiel (Quadrant III) pareto-inferior ist: Beide Spieler könnten sich besserstellen, wenn beide die Waffen ruhen lassen würden. Quadrant I bietet eine Friedensdividende, die den Parteien durch (Bürger-)Krieg verloren geht.

[27] In diesem Kontext kann man nun zwei Konzepte von Pazifismus unterscheiden. Das erste Konzept fasst Pazifismus als Ziel auf, nach Frieden zu streben, also Quadrant I zu erreichen (Win-Win). Das zweite Konzept fasst Pazifismus als Mittel auf, und zwar als unkonditionierte Waffenruhe. Machte Partei A sich das zweite Konzept zu eigen, würde Quadrant I verfehlt und stattdessen Quadrant IV realisiert (Win-Lose). Für Partei B wäre dies der `best case`, für Partei A hingegen der 'worst case $(A$ bb. 3). Eine Ziel-Mittel-Konfusion liegt dann vor, wenn das Mittel normativ überhöht wird, so dass man es als Selbstzweck (= Ziel) betrachtet. Eine solche Konfusion ist im vorliegenden Fall besonders misslich, weil das pazifistische Mittel ungeeignet ist, das pazifistische Ziel zu erreichen. Hier bleibt man dem Trade-off-Denken verhaftet. Man befindet sich gleichsam in einem Gedankengefängnis. Aus ihm kann man mittels einer orthogonalen Positionierung ausbrechen, graphisch: durch einen Wechsel der Denkrichtung um 90 Grad. Das Motto lautet: sthinking outside the box'.

[28] Es ist genau diese Problemsituation, die Hobbes mit seiner Theorie zu bearbeiten versucht. Seine Frage lautet: Wie kann ein (Bürger-)Krieg durch einen Friedensschluss beendet werden? Den Bürgerkrieg fasst Hobbes als ein soziales Dilemma auf: als einen Krieg aller gegen alle, der zu einer wechselseitigen Schlechterstellung führt. Im Zustand des Krieges ist gemäß Hobbes (1996 [1651]: 84) das Leben des einzelnen "solitary, poor, nasty, brutish, and short « - und damit für alle Menschen gleichermaßen unerträglich.

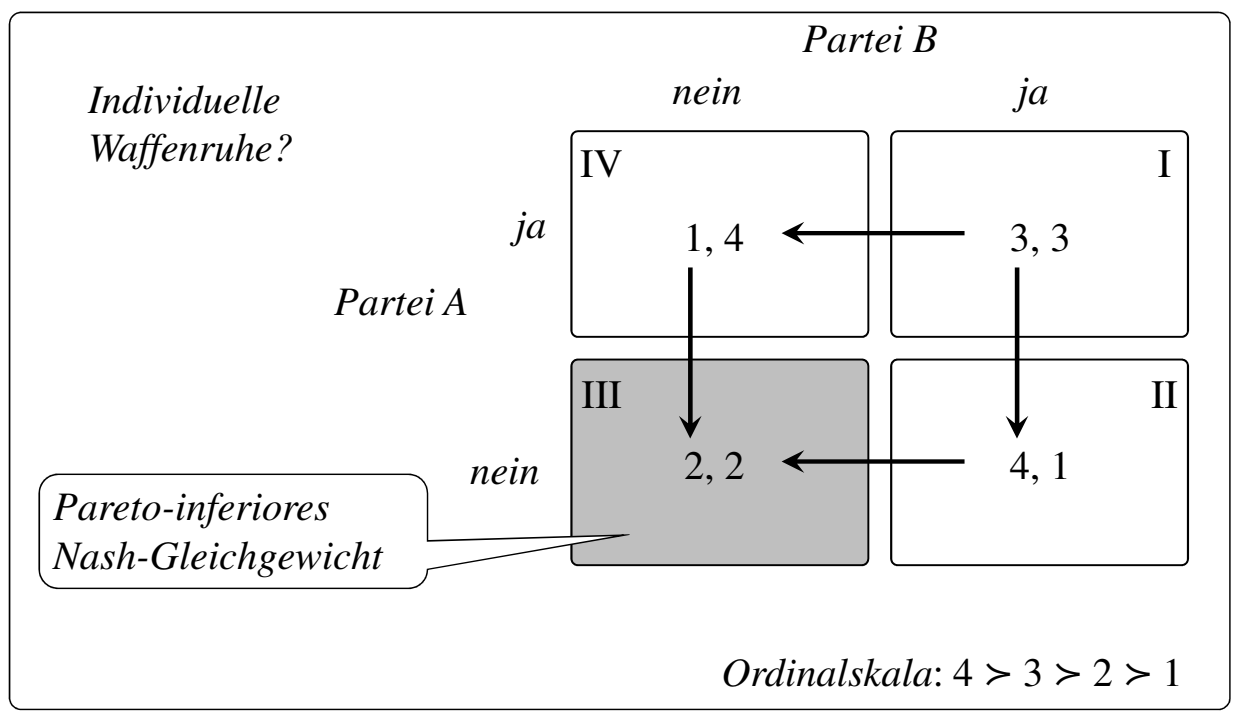

Abbildung 2: Das (Bürger-)Kriegsdilemma (Quelle: eigene Darstellung) 
[29] Hobbes argumentiert nun, dass man aus dem Kriegszustand herauskommen kann, indem man sich an das Naturrecht hält. Das Naturrecht formuliert Regeln der Vernunft, denen sich Rechte und Pflichten entnehmen lassen. Über die beiden obersten Naturrechtsregeln schreibt Hobbes (1996 [1651]: 87, H.i.O.):

»[I]t is a precept, or general rule of reason, that every man, ought to endeavour peace, as far as he has hope of obtaining it; and when he cannot obtain it, that he may seek, and use, all helps, and advantages of war. The first branch of which rule, containeth the first, and fundamental law of nature; which is, to seek peace, and follow it. The second, the sum of the right of nature; which is: by all means we can, to defend ourselves.

From this fundamental law of nature, by which men are commanded to endeavour peace, is derived this second law; that a man be willing, when others are so too, as far-forth, as for peace, and defence of himself he shall think it necessary, to lay down this right to all things; and be contented with so much liberty against other men, as he would allow other men against himself. For as long as every man holdeth this right, of doing anything he liketh; so long are all men in the condition of war. But if other men will not lay down their right, as well as he; then there is no reason for anyone to divest himself of his: for that were to expose himself to prey, (which no man is bound to) rather than to dispose himself to peace."

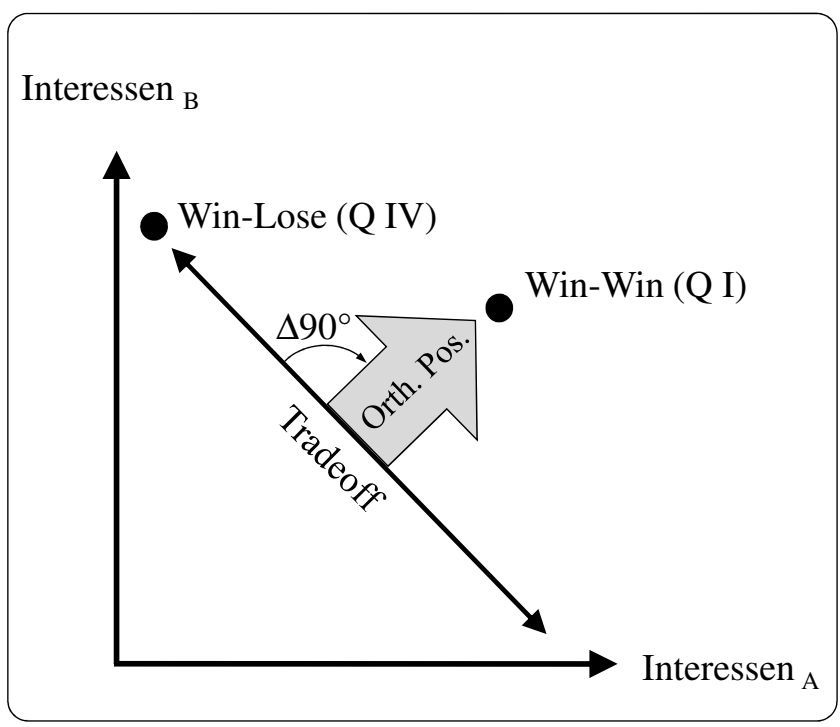

Abbildung 3: Orthogonale Positionierung zur Auflösung der Ziel-Mittel-Konfusion (Quelle: eigene Darstellung)

[30] Die erste Naturrechtsregel nimmt Hobbes in zwei Teile auseinander. Der erste Teil erklärt die Friedenssuche zur Vernunftpflicht. Damit wird Quadrant I normativ ausgezeichnet. Der zweite Teil definiert das Notrecht, im Kriegszustand alle Mittel gebrauchen zu dürfen, die zur Selbsterhaltung nötig sind. Damit wird Quadrant IV normativ abgelehnt.

[31] Aus der so sezierten ersten Naturrechtsregel leitet Hobbes sodann die zweite Naturrechtsregel ab. Sie formuliert die - bedingte! - Pflicht zum Friedenswillen und benennt als Bedingung, dass alle anderen ebenfalls den Frieden wollen 
müssen, bevor sich dann alle auf ein System gleicher Freiheiten einigen können, das den Krieg überwindet.

[32] Hobbes' spezifischer Erkenntnisbeitrag lässt sich noch deutlicher herausarbeiten, wenn man folgende Textpassage genau studiert (Hobbes 1996 [1651]: 105, H.i.O.):

"The laws of nature oblige in foro interno; that is to say, they bind to a desire they should take place: but in foro externo; that is, to the putting them in act, not always. For he that should be modest, and tractable, and perform all he promises, in such time, and place, where no man else should do so, should but make himself a prey to others, and procure his own certain ruin, contrary to the ground of all laws of nature, which tend to nature's preservation. And again, he that having sufficient security, that others shall observe the same laws towards him, observes them not himself, seeketh not peace, but war; and consequently the destruction of his nature by violence."

[33] Hier argumentiert Hobbes, dass alle Naturrechtsregeln dem Ziel dienen, Frieden zu schaffen, und dass der Friede seinerseits ein Mittel ist, das der Erhaltung des eigenen Lebens dient. Hinsichtlich der Geltung von Naturrechtsregeln trifft Hobbes nun eine merkwürdige - und vor allem: denkwürdige! - Unterscheidung: Aus seiner Sicht gelten diese Regeln ausnahmslos immer in foro interno; aber sie gelten nicht immer ausnahmslos in foro externo. Wie ist das zu verstehen?

[34] Um einen Deutungsvorschlag zu entwickeln, interpretiere ich das Gefangenendilemma aus Abbildung 2 als Basisspiel und ordne dieser ersten Arena mit Hilfe des ordonomischen Drei-Ebenen-Schemas zwei weitere Arenen zu: die Arena kollektiver Friedensverhandlungen (Ebene 2) sowie die Arena öffentlicher Meinungsbildung (Ebene 3). Abbildung 4 vermittelt einen Überblick. Auf der linken Seite ist das Problem abgebildet, auf der rechten Seite die Lösung: Das Problem besteht in einem durch Diskursversagen blockierten Lernprozess, die Lösung hingegen in einer orthogonalen Positionierung, die die pazifistische Ziel-MittelKonfusion überwindet und so gedanklich den Weg frei macht, damit ein im allgemeinen Interesse liegender funktionaler Friedensschluss organisiert werden kann.

[35] Links unten abgebildet ist das dilemmatische Basisspiel. Das Nash-Gleichgewicht (= der schwarze Punkt) ist pareto-inferior. Die Friedensdividende kann nicht angeeignet werden. In der Mitte links ist das zugehörige Metaspiel abgebildet. Die politischen Friedensverhandlungen misslingen, weil das Metaspiel die dilemmatische Struktur des Basisspiels dupliziert. Letztlich verantwortlich hierfür ist das Meta-Metaspiel auf Ebene 3, das vom Trade-off-Denken dominiert wird. Die Parteien nehmen sich als Feinde wahr, so dass ihnen das gemeinsame Interesse am Frieden aus dem Blick gerät.

[36] Die Veränderung des 'mental model ist der Schlüssel zum Erfolg, graphisch repräsentiert durch die rechte Seite von Abbildung 4, die von oben nach unten zu lesen ist. Erst die orthogonale Positionierung zu diesem eindimensional auf Konflikt verengten Wahrnehmungsrahmen im diskursiven Meta-Metaspiel eröffnet die Option, das politische Metaspiel mit Hilfe einer bedingten Friedensstrategie aus einem Dilemmaspiel in ein reines Koordinationsspiel zu verwandeln, so dass der gemeinsame Friedensschluss dann schließlich auch das Basisspiel 
transformiert, mit der Folge, dass Quadrant I sich als neues Gleichgewicht mit Friedensdividende einstellt. ${ }^{10}$

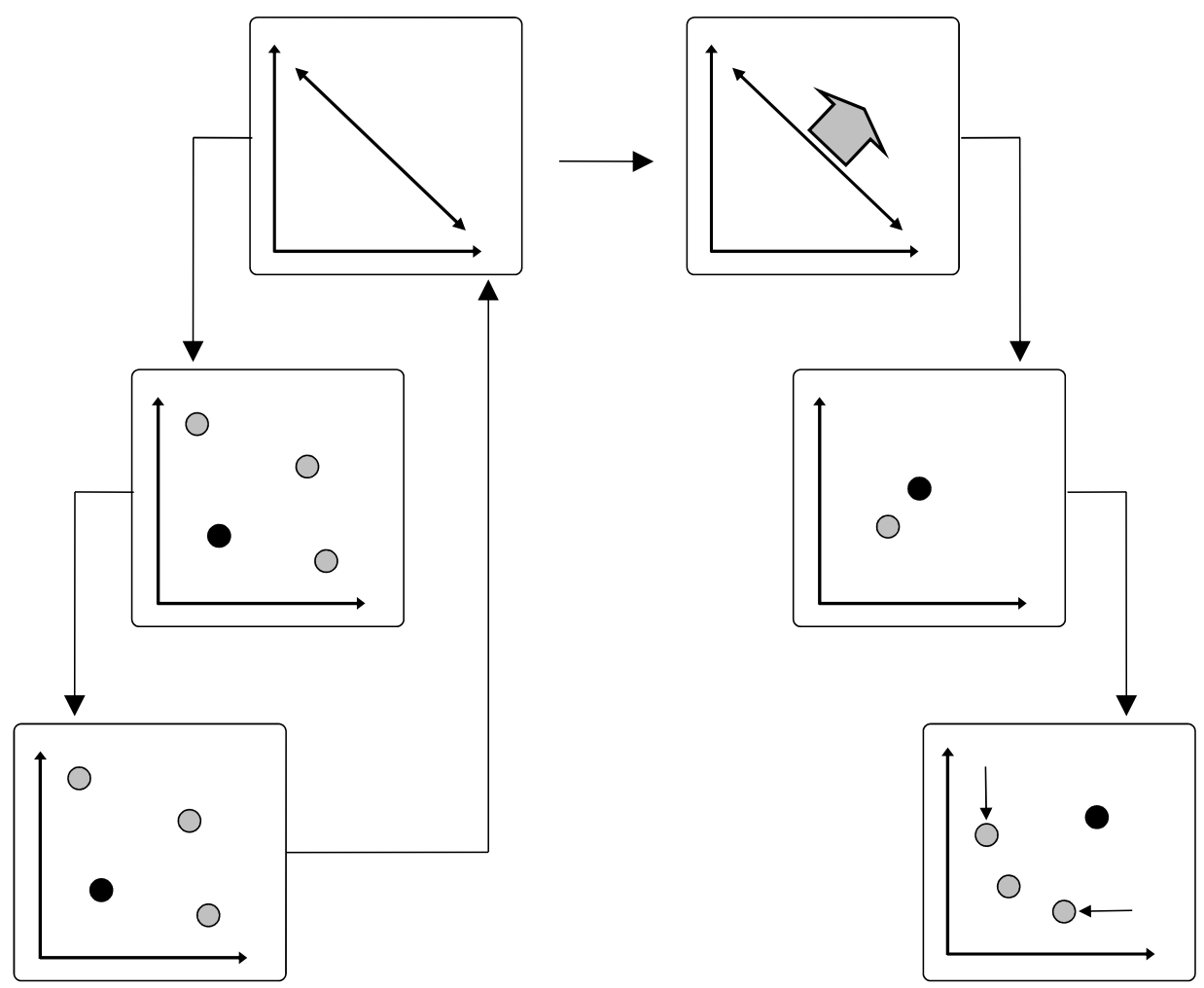

Abbildung 4: Das ordonomische Drei-Ebenen-Schema (Quelle: modifiziert in Anlebnung an Pies [2012c: Abb. 7, S. 30])

10 Zur Erläuterung: Wenn beide Parteien mit der bedingten Kooperations-Strategie >ich rüste nur dann $a b$, wenn auch du abrüstest $<$ in die Verhandlungen gehen, nehmen sie im Hinblick auf das Gefangenendilemma in Abbildung 2 - die Quadranten II und IV aus dem Spiel. Deshalb sind in Abbildung 4 Mitte rechts nur noch die Payoff-Paare $(2,2)$ und $(3,3)$ eingezeichnet. Folglich liegt hier kein Konflikt mehr vor, sondern reine Interessenharmonie. Die Verhandlung hat sich vom Dilemma in ein reines Koordinationsspiel mit neuem Gleichgewicht (= schwarzer Punkt) verwandelt. Dieses Metaspiel hat Folgen für das Basisspiel in Abbildung 4 unten rechts: Wenn im Metaspiel eine geeignete Sanktionsstruktur vereinbart wird, verlieren (symbolisiert durch die beiden Pfeile) im Basisspiel die Quadranten II und IV ihre Attraktivität, was dann zur Konsequenz hat, dass das Payoff-Paar $(3,3)$ zum Nash-Gleichgewicht (= schwarzer Punkt) wird, so dass das ursprüngliche Dilemma durch eine Reformierung des Denkens und Handelns überwunden ist. Die Institutionalisierung der Friedenslösung im Metaspiel ermöglicht es, die Friedensorientierung vom forum internum (= Denken) auf das forum externum (= Handeln) so zu übertragen, dass eine dysfunktionale Ziel-Mittel-Konfusion vermieden und der gesuchte Frieden tatsächlich verwirklicht wird. 
[37] Gestützt auf diese Darstellung lässt Hobbes sich nun so deuten, dass er eine für die Reflexion (und Implementierung) von Moral enorm bedeutsame Distinktion einführt, indem er Denken und Handeln konzeptionell entkoppelt und zugleich institutionell miteinander verknüpft: Bei Hobbes gelten die Naturrechtsregeln stets für das Denken (= in foro interno; >desire<; Friedenssehnsucht), nicht aber stets für das Handeln (= in foro externo; 'putting in act<, Friedfertigkeit). Hier führt er eine Konditionierung ein, die der pazifistischen Ziel-Mittel-Konfusion konzeptionell ein Ende bereitet.

[38] Zur Erläuterung: Befindet sich die Gesellschaft in einer anarchischen Situation, d.h. im sozialen Dilemma des (Bürger-)Krieges - vgl. das Basisspiel in Abbildung 4 unten links -, dann kann es um der Selbsterhaltung des einzelnen Individuums willen nicht zur Pflicht erklärt werden, den Frieden zu halten, solange die anderen dazu nicht auch bereit sind. Dies hieße andernfalls, den einzelnen zum Opfer zu machen. Dabei würde dann das Ziel dem Mittel geopfert. Das wäre widersinnig. Befindet sich die Gesellschaft hingegen in einer Situation, in der das soziale Dilemma bereits überwunden ist - vgl. das Basisspiel in Abbildung 4 unten rechts -, dann entspricht es Hobbes zufolge nicht nur der Pflicht, sondern auch der Klugheit (insbesondere dem Interesse an Selbsterhaltung), sich an das sanktionsbewehrte Gesetz zu halten und den bereits erreichten Frieden nicht zu brechen. Hier ist das Mittel dem Ziel dienlich.

[39] Unbeschadet dessen, was - je nach Kondition - auf der Handlungsebene des Basisspiels gilt, kann Hobbes so interpretiert werden, dass er für die Ebene des Meta-Metaspiels - vgl. die obere Spielebene in Abbildung 4 - die unbedingte Pflicht ausgibt, stets den Frieden suchen zu sollen. Dies entspricht der Aufforderung, im öffentlichen Diskurs seinen eigenen Friedenswunsch zu bekunden und für Win-Win-Optionen aufgeschlossen zu sein. Die individuelle $>$ Fried-Fertigkeit $<-$ der Wunsch zum Frieden, die Friedensbereitschaft: das Geneigtsein, den Kriegszustand $\mathrm{zu}$ verlassen - liegt immer im eigenen Interesse an Selbsterhaltung, und zwar völlig unabhängig davon, ob noch Krieg herrscht oder bereits Friede geschlossen wurde.

[40] Mit seiner hochgradig differenzierten Naturrechtsargumentation markiert Hobbes folglich einen Hiatus zwischen Denken und Handeln, der erst durch die Schaffung eines institutionellen Arrangements allgemeiner Sicherheit (und damit: begründeter Sicherheitserwartung) - vgl. das Metaspiel in Abbildung 4 Mitte rechts - überwunden werden muss, bevor der Weg vom Denken zum Handeln, vom forum internum zum forum externum, vom Meta-Metaspiel zum Basisspiel, vom Friedenswollen zum Friedenskönnen erfolgreich beschritten werden kann. Insofern arbeitet Hobbes mit einem Ansatz, der Normativität als bedingtes Sollen auffasst und dann - als Theorie - dazu beitragen will, diese Bedingungen in der gesellschaftlichen Praxis aktiv herzustellen.

[41] Demgegenüber würde der Verzicht auf eine institutionelle Vermittlung von Denken und Handeln - als pazifistische Ziel-Mittel-Konfusion - das Problem nicht lösen, sondern verschärfen, weil vom Friedenswillen (im Denken) unvermittelt auf eine Friedenspflicht (im Handeln) geschlossen würde, mit der Folge, ein Mittel zu propagieren, das die Zielerreichung vereitelt, solange der Konflikt noch 
nicht verlässlich überwunden ist. Deshalb kulminiert die Pointe der Hobbesschen Überbietungsargumentation in der Erkenntnis, dass es unmoralisch wäre, die Waffen ruhen zu lassen, solange nicht institutionell sichergestellt ist, dass sich auch die andere Seite an der Waffenruhe beteiligt.

[42] Trotz manch gravierender Unterschiede - deutlich ablesbar an ihrer demokratischen Konsensorientierung und am Verzicht auf naturrechtliche Begründungen - steht die Ordonomik dem Hobbesschen Ansatz (in der hier vorgestellten Lesart) methodologisch in mehrerlei Hinsicht besonders nahe: Zum einen betont Hobbes wie nur wenige Ethiker vor ihm (und nach ihm) den spezifisch moralischen Stellenwert des Eigeninteresses. Zum anderen betont Hobbes wie nur wenige Ethiker vor ihm (und nach ihm) die besondere Situationseigenschaft des Wettbewerbs, eigeninteressierte Akteure dem Druck (und sogar dem Zwang) auszusetzen, einer Gefährdung der eigenen Existenz durch Konkurrenten proaktiv entgegenzuwirken. Drittens ist Hobbes die konzeptionelle Pionierleistung zuzurechnen, für eine nicht nur in seinem Jahrhundert eminent wichtige Ziel-MittelKonfusion das passende Überbietungsargument generiert zu haben. Und viertens schließlich entwickelt Hobbes mit seiner Distinktion von forum internum und forum externum ein bis heute kaum rezipiertes, aber hoch leistungsfähiges Modell für gesellschaftliche Lernprozesse zur Verwirklichung von Normativität im Modus institutioneller Reformen.

\section{Wie lässt sich eine orthogonale Positionierung zur Flüchtlings(politik)debatte erarbeiten? ${ }^{11}$}

[43] Wir wissen nun, was wir nach ordonomischem Verständnis (a) unter einer den Diskurs blockierenden Ziel-Mittel-Konfusion zu verstehen haben, (b) welche diskursive Qualität Überbietungsargumente aufweisen und (c) welch bedeutende Rolle institutionelle Reformvorschläge zur besseren Verwirklichung berechtigter normativer Anliegen spielen. Solchermaßen ausgestattet mit dem ordonomischen Konzept einer >orthogonalen Positionierung nehme ich nun die eingangs dargelegte Diagnose wieder auf, dass die zeitgenössische Flüchtlings(politik)debatte unter Diskursblockaden leidet.

[44] Metaphorisch ausgedrückt lautet der empirische Ausgangsbefund, dass der demokratische Gesprächsfaden verknotet ist. Allerdings handelt es sich nicht um einen gordischen Knoten, der eines jungen Alexanders bedürfte, um mit der Entschlossenheit eines mutigen Schwerthiebs durchtrennt zu werden. Vielmehr handelt es sich um zahlreiche dezentral geknüpfte Knoten, die massenmedial festgezurrt wurden - und nun durch mühselige Aufklärungsarbeit wieder aufgedröselt werden müssen.

11 Die zeitgenössisch aktuelle Debatte zur Flüchtlingspolitik weist strukturelle Analogien auf mit der Debatte zur Asylpolitik, die in der ersten Hälfte der 1990er Jahre in Deutschland geführt wurde. Vgl. hierzu Pies (2015 [1993]). Anregungen für eine langfristig orientierte Migrationspolitik finden sich bei von Weizsäcker (2016). Vgl. hierzu auch die Videodokumentation im Internet unter www.hafop.de. 
[45] Ordonomisch betrachtet liegt die Ursache des Problems in einer dysfunktionalen (Ver-)Wendung der Moralkommunikation. Wir haben es mit einem moralisch radikalisierten Meinungsspektrum zu tun, innerhalb dessen sich zwei Seiten gegenüberstehen, die sich selbst als moralisch gerecht(fertigt) empfinden und die jeweilige Gegenseite als unmoralisch abstempeln. ${ }^{12}$ Dies wiederum hat einen tieferen Grund darin, dass die Sachauseinandersetzung eindimensional verengt worden ist und letztlich auf die rein quantitative Frage zugespitzt wurde, wie viele Flüchtlinge auf deutschem Boden aufgenommen werden sollen. Es ist diese Verengung, die für eine strikte Entgegensetzung der Positionen sorgt. Legt man die radikalen Enden des Meinungsspektrums zugrunde, ist die Anzahl der in Deutschland aufgenommenen Flüchtlinge den einen zu niedrig und den anderen zu hoch. Während die eine Seite in einer akuten Notsituation der humanitären Herausforderung mit einer Willkommenskultur für möglichst viele Flüchtlinge begegnen möchte und in dieser Hinsicht auf >Alternativlosigkeit< pocht, sorgt sich die andere Seite um einen möglichen Kontroll- und Souveränitätsverlust, um die Preisgabe staatlicher Grenzsicherung und eine Überforderung des Gemeinwesens.

[46] Auf der Suche nach einem Überbietungsargument, das diese polemogene, den Diskurs auf Streiteskalation programmierende Frontstellung überwindet, geht die Ordonomik so vor, dass sie beide Positionen - und vor allem: die moralischen Anliegen, die beiden Positionen zugrunde liegen - ernst nimmt und zu überbieten versucht. Das betrifft zum einen das Anliegen der Pro-Seite, großzügig humanitäre Hilfe zu leisten. Und es betrifft spiegelbildlich die Contra-Seite und deren Sorge um das nationale Interesse, d.h. um das Gemeinwohl der einheimischen Bevölkerung. Im Prinzip lässt sich die orthogonale Positionierung in einem Dreischritt entwickeln, indem zunächst beide Positionen dekonstruiert und sodann im Zusammenhang rekonstruiert (und argumentativ überboten) werden. Wie das

12 Man kann diese ordonomische Diagnose mit einer hellsichtigen Formulierung von Niklas Luhmann (1990: 23f.) weiter präzisieren: »Die Funktionssysteme verdanken ihre Autonomie einer jeweils verschiedenen Funktion, aber auch einer je besonderen binären Codierung, also zum Beispiel der Unterscheidung von wahr und unwahr im Falle des Wissenschaftssystems oder der Unterscheidung von Regierung und Opposition im demokratischen politischen System. In keinem dieser Fälle können die beiden Werte dieser Codes mit den beiden Werten des Moralcodes kongruent gesetzt werden. Es darf gerade nicht dahin kommen, dass man die Regierung für strukturell gut, die Opposition für strukturell schlecht oder gar böse erklärt. Das wäre die Todeserklärung für Demokratie. "Das zeitgenössische Problem ist etwas anders gelagert: Hier stehen sich nicht Regierung und Opposition gegenüber, sondern Regierung und beträchtliche Teile der Bevölkerung. Und es ist nicht einfach nur die Regierungsseite, die kritische Bevölkerungsgruppen als unmoralisch abwertet. Vielmehr kommt verschärfend hinzu, dass kritische Bevölkerungsgruppen auch umgekehrt die Regierung und ihre Anhänger für strukturell schlecht oder gar böse erklären. Auch wenn man gegenwärtig noch nicht von einem >Todesurteil< sprechen mag: Inwiefern die Demokratie diese Krise verarbeiten kann, wird davon abhängen, ob - und auch: wie schnell - es gelingt, diese unselige Frontstellung zu überwinden. Falls es nicht schnell gelingt, drohen jedenfalls tektonische Verschiebungen im Parteiengefüge sowie forciert populistische und sogar autoritäre Entwicklungstendenzen. 
gehen kann, will ich nun zumindest in den Grundzügen vor Augen führen. Dabei soll deutlich werden, wie wichtig es ist, die >richtigen Fragen zu stellen.

[47] Erster Schritt: Zur Aufsprengung des Denkrahmens der Pro-Seite lautet die entscheidende Frage, wem nicht geholfen wird, wenn man in Deutschland großzügig (und sogar großherzig) Flüchtlinge aufnimmt. Mit dieser Frage rücken drei Gruppen von Menschen ins Blickfeld, die in der gegenwärtigen Diskussion kaum eine Rolle spielen: (a) jene Flüchtlinge, denen es zwar gelingt, eine Krisenregion (z.B. ein Bürgerkriegsgebiet) zu verlassen, die es aber nicht schaffen, ihren Fuß auf europäischen oder gar deutschen Boden zu setzen; (b) jene Flüchtlinge, die innerhalb einer Krisenregion Haus und Hof verlassen, um ihr Leben zu retten, es aber nicht ins benachbarte Ausland schaffen; sowie schließlich (c) jene Menschen, die sich in Not befinden, ohne Flüchtlinge zu sein (z.B. weil sie aufgrund von Krankheit oder Hunger nicht mehr mobil sind). Rückt man diese drei Gruppen ins Zentrum der Betrachtung, dann wird der Blick dafür frei, dass sich das moralische Anliegen, humanitäre Hilfe zu leisten, nicht darauf beschränken lässt, diese Hilfe ausschließlich auf deutschem Boden zu leisten. ${ }^{13}$

[48] Die argumentative Stoßrichtung dieses ersten Schritts zielt darauf, das Anliegen humanitärer Hilfe besser zu verwirklichen, indem man sich bewusst macht, dass man größere Not lindern kann, wenn man nicht (nur) im Inland, sondern (auch) im Ausland Hilfe leistet. Denn es zeugt von einer moralischen ZielMittel-Konfusion, wenn man übersieht, dass das humanitäre Anliegen darin besteht, auch jenen Menschen zu helfen, die es auf ihrer Flucht nicht bis nach Deutschland schaffen, sowie die Lebensumstände auch solcher Menschen zu verbessern, die sich in Not befinden, ohne Flüchtlinge zu sein. Um es so klar wie möglich zu formulieren: Die humanitäre Hilfe auf deutschem Boden ist nur ein Mittel - und zudem nur ein Mittel neben alternativen anderen Mitteln -, um das moralische Ziel zu verfolgen, weltweit möglichst vielen Menschen wirksam zu helfen. In diesem Kontext ist daran zu erinnern, dass vergleichsweise arme Länder wie der Libanon oder Jordanien pro Kopf ihrer Bevölkerung wesentlich mehr Flüchtlinge aufgenommen haben als Deutschland, und dass viele dieser Flüchtlinge dort unter prekären Umständen leben - was nahelegt, dass man wesentlich mehr Menschen wirksam helfen kann, wenn man die Hilfe nicht daran knüpft, dass sie auf deutschem Boden geleistet und empfangen werden muss. Die - für diese und ähnliche Situationen - >richtige< Frage beherzigt das ökonomische Prinzip und lautet in der schönen Formulierung von William MacAskill (2015: 10f.): «How can we ensure that we avoid accidentally causing harm, and succeed in having the greatest positive impact we can? « Wer so fragt, wird gerade aus moralischen Gründen die Hilfe nicht ausschließlich den jungen, starken, mobilen

13 Um Missverständnissen vorzubeugen, sei hier auf einen Aphorismus verwiesen, mit dem Marie von Ebner-Eschenbach (1939 [1880]: 19) einen naheliegenden Fehler im Denken und Handeln markiert: » Man kann nicht allen helfen! sagt der Engherzige und - hilft keinem. «Im Klartext: Mir geht es nicht darum, weniger Hilfe zu leisten, sondern darum, die Hilfe effektiver werden zu lassen, d.h. mehr Menschenleben zu retten. 
und gesunden Menschen zukommen lassen wollen, sondern auch alten, schwachen, mobilitätsbeeinträchtigten und kranken.

[49] Zweiter Schritt: Zur Aufsprengung des Denkrahmens der Contra-Seite lautet die entscheidende Frage, ob man das nationale Interesse unter der utopischen Annahme nationaler Isolation oder unter der realistischen Annahme global zunehmender Interdependenzen diskutieren möchte. Letzteres schließt aus, sich mit dem frommen Wunsch zufrieden zu geben, Deutschland möge von internationalen Entwicklungen unabhängig sein. Vielmehr ist das genaue Gegenteil der Fall. Angesichts zunehmender Interdependenzen wird die Gestaltung internationaler Entwicklungen zu einer ordnungspolitischen Aufgabe, die Deutschland freilich am besten im Konzert der europäischen Mitgliedsstaaten angehen kann. Und das wiederum bedeutet, dass es im nationalen Interesse liegt, sich international nicht weniger, sondern mehr zu engagieren. Für ein verstärktes Engagement gibt es unabweisbare Klugheitsargumente: Mit Blick auf die Zukunft der nächsten Jahrzehnte besteht im Kontext der Flüchtlingspolitik ein nationales Interesse daran, sich im Rahmen der Europäischen Union sehr viel stärker und sehr viel systematischer als bisher - d.h. mit sehr viel mehr Ressourcen sowie mit einer auf Rechtsprinzipien basierenden Langfristorientierung - pro-aktiv für internationale Konfliktlösungen einzusetzen, um insbesondere im arabischen Raum und auf dem afrikanischen Kontinent Krisenprävention - und falls nötig: Krisenmanagement zu fördern und gegebenenfalls selbst aktiv zu betreiben.

[50] Ähnlich wie dem radikalen Spektrum der Pro-Seite, liegt also auch dem radikalen Spektrum der Contra-Seite eine Ziel-Mittel-Konfusion zugrunde, was sich an der symbolpolitischen Beschwörung einer >Obergrenze` für Flüchtlinge auf deutschem Boden leicht beobachten lässt. Hier wird dann so getan, als sei dem nationalen Interesse schon bestens damit gedient, die Mauern um die Festung Europa möglichst hochzuziehen. Mauern aber sind kein Ziel, sondern allenfalls ein Mittel der Politik.

[51] Der hier einschlägigen Konfusion von Zielen und Mitteln wird durch die viel bemühte Semantik gemeinsamer Werte Vorschub geleistet. Sie erweist sich nach wie vor als eine schwere Hypothek für die öffentlichen Diskurse der demokratischen Öffentlichkeit. Bei der aktuellen Flüchtlings(politik)debatte zeigt sich dies freilich mit besonderer Deutlichkeit und Dringlichkeit: Der Unterschied zwischen den Denkkategorien >Wertekonsens` und 'Regelkonsens k kommt dem Unterschied zwischen einer Exklusionssemantik und einer Inklusionssemantik gleich: Die Berufung auf (und die Beschwörung von) gemeinsame(n) Werte(n) grenzt heterogene Bevölkerungselemente aus. Sie wirkt desintegrierend. Demgegenüber schließt die Betonung von Regelkonsens heterogene Bevölkerungselemente integrativ ein. Insofern rückt das Motto >Regelkonsens statt Wertekonsens` (vgl. hierzu allgemein Pies [2012a sowie insbesondere 2012b und 2016c]) völlig andere Gesichtspunkte ins Zentrum einer aufklärenden Debatte zur kollektiven Selbstverständigung darüber, wie ein angemessenes Verständnis von nationalem Interesse auszusehen hat, das dem Kontext global zunehmender Interdependenzen intellektuell Rechnung trägt. Wer so fragt, wird unweigerlich zu der Erkenntnis gelangen, dass es im nationalen Interesse liegt, sich international stärker zu engagieren. 
[52] Dritter Schritt: Hier lautet die systematische Frage, ob sich inmitten all des Streits diskursive Konsenszonen auffinden lassen, wenn man den aktuellen Status quo durch eine Brille betrachtet, in die die ordonomisch geschärften Gläser eines aufgeklärten humanitären Hilfeanliegens und eines aufgeklärten nationalen Interesses eingesetzt sind. Aus Platzgründen möge ein einziges Beispiel als illustrativer Hinweis genügen, wie man orthogonale Positionierungen in institutionelle Reformvorschläge umsetzen kann.

[53] Nach Schließung der Balkanroute ist - wie zu erwarten war - die Mittelmeerroute wieder reaktiviert worden. Die Mitgliedsstaaten der Europäischen Union reagieren darauf mit organisierten Rettungsaktionen. Sie lassen Flottenverbände im Mittelmeer patrouillieren, die in Seenot geratene Flüchtlinge aufgreifen (sollen), um sie sodann auf europäischem Boden in Sicherheit zu bringen. Trotz intensivierter Bemühungen ertrinken dennoch zahlreiche Menschen, die nach der Havarie ihrer Boote nicht rechtzeitig gefunden werden.

[54] Diese Rettungsbemühungen der Europäischen Union setzen eine nichtintendierte Entwicklung in Gang: Je intensiver man versucht, in Seenot geratene Flüchtlinge rechtzeitig aufzugreifen, umso schlechter ist die Qualität der Boote (und die Ausstattung mit Wasservorräten), die oft nur dazu ausreicht, vom afrikanischen Festland aus gerade noch internationale Gewässer zu erreichen. Angesichts dieser Lage ist es wenig hilfreich, die Schuld gewissenlosen Schlepperbanden zuzuweisen, die verzweifelte Menschen in überfüllten Booten auf eine hoch riskante Reise schicken. Noch weniger hilfreich aber ist es, die Augen davor zu verschließen, dass gerade die Aussicht, aus Seenot gerettet zu werden und so Europa als Flüchtling zu erreichen, viele Menschen allererst mit dem Anreiz ausstattet, sich in große Gefahr zu begeben, d.h. ihr Leben zu riskieren. ${ }^{14}$

[55] Die ordonomische Überbietungsargumentation lautet: Es ist weder mit dem Anliegen humanitärer Hilfe vereinbar, noch liegt es im nationalen Interesse Deutschlands, an einem Status quo festzuhalten, der in den letzten Jahren tausende von Flüchtlingen das Leben gekostet hat und erwarten lässt, dass auch in Zukunft zahlreiche Menschen bei dem Versuch sterben werden, als Flüchtling nach Europa zu gelangen. Insofern lässt sich hier ein gemeinsames Reformanliegen identifizieren, das bei einer nicht rückwärts, sondern vorwärts gewandten Diskussion der sachlichen Zusammenhänge allen Beteiligten die Augen öffnen könnte, wie man aus der Diskursblockade wieder herausfinden kann - indem

14 Die dilemmatische Logik dieser Situation ist vergleichbar mit dem (nicht gewinnbaren) Drogenkrieg, den reiche Demokratien zu führen versucht haben, ohne der Tatsache Rechnung zu tragen, dass die inländische Nachfrage nach Drogen ein ausländisches Angebot erzeugt. Vgl. hierzu Hartwig/Pies (1995). Eine ähnliche Situationslogik trat auch Mitte der 1990er Jahre auf, als zivilgesellschaftliche Organisationen sich bemühten, Spendengelder zu sammeln, um im Sudan entführte und versklavte Bürger freizukaufen. Das zusätzliche Lösegeld führte offenbar zu zusätzlichen Entführungen. Vgl. Miller et al. (2005: 53-56). Eine vergleichbare Interaktionsdynamik war auch in Zeiten des Kalten Krieges zu beobachten, als die humanitäre Strategie Westdeutschlands, politische Gefangene der DDR freizukaufen, für das ostdeutsche Regime einen Anreiz schuf, mehr Inhaftierungen vorzunehmen, um Devisen zu beschaffen. 
man sich mit dem Auffinden einzelner Konsenszonen schrittweise neue Gemeinsamkeiten erarbeitet, die als Zustimmungs- und Verständigungsbasis und damit als Ausgangspunkt für konstruktive Lernprozesse fungieren können: Ein beiden Seiten gemeinsames Anliegen besteht perspektivisch darin, die Mittelmeerroute zu schließen und gleichzeitig - als Junktim! - ein geordnetes Verfahren zu etablieren, das politisch Verfolgten Asyl bietet, Flüchtlingen das Leben rettet sowie für Kontingente von Arbeitsmigranten und ihre Familien eine legale Option eröffnet, ohne Lebensgefahr nach Europa zu kommen.

- Die Mittelmeerroute lässt sich am einfachsten dadurch schließen, dass man aufgegriffene Flüchtlinge nicht nach Europa, sondern in ihr Heimatland zurückbringt bzw. in Auffanglager auf dem afrikanischen Kontinent, in denen die EU einen hohen Standard humanitärer Versorgung garantiert (und finanziert). ${ }^{15}$

- Geordnete Verfahren lassen sich relativ leicht dadurch einrichten, dass man die Möglichkeit eröffnet, nicht erst auf europäischem Boden eine (befristete oder dauerhafte) Aufenthaltsgenehmigung als Asylant, als Flüchtling oder als Erwerbsperson zu erhalten. Hierzu könnte die EU über die Botschaften ihrer Mitgliedsländer ein globales Netzwerk von Außenstellen einrichten, das besonders in den relevanten Krisenregionen allen Menschen den Zugang eröffnet, sich für einen legalen Aufenthaltsstatus (explizit auch als Arbeitsmigrant) zu bewerben.

\section{Reflexion und »lessons (to be) learned «}

[56] Im Anschluss an das hier zur Illustration der ordonomischen Methode entwickelte Anwendungsbeispiel der Flüchtlings(politik)debatte möchte ich nun versuchen, mir gewissermaßen selbst über die Schulter zu schauen bzw. schauen zu lassen. Als Reflexion zur ordonomischen Methode sind sieben Beobachtungen zu notieren:

[57] Erstens: Das Denken in dualistischen Werte-Trade-offs kann fundamentalistische Züge annehmen und die Moralkommunikation als Streiteskalation entgleisen lassen. Diskursblockaden bis hin zum Diskursversagen sind die Folge. Für demokratische Gesellschaften ist das eine arge Verlegenheit.

[58] Zweitens: Nicht immer ist das Trade-off-Denken auf Ziel-Mittel-Konfusionen zurückzuführen. Wohl aber gilt das Umgekehrte: Ziel-Mittel-Konfusionen legen immer die Tendenz nahe, in dualistischen Trade-offs (insbesondere in den

15 Australien praktiziert eine ähnliche Lösung. Entsprechende Vorschläge für Europa liegen seit langem auf dem Tisch. Erinnert sei nur an die Initiative von Otto Schily aus dem Sommer 2004. Vgl. FAZ (2004). Erinnert sei aber auch daran, dass solche Vorschläge über Jahre hinweg gezielt diskreditiert wurden - i.d.R. mit fadenscheinigen Argumenten, die den Eindruck erwecken sollten, als läge einer geordneten Schließung der Mittelmeerroute nicht ein humanitäres Anliegen - oder als läge sogar ein nichthumanitäres Anliegen - zugrunde. 
Kategorien von Gut und Böse und damit letztlich in Freund-Feind-Schemata) zu denken - und so tribalistische Emotionslagen zu aktivieren.

[59] Drittens: Die aktuelle Flüchtlings(politik)debatte ist dafür ein empirisches Beispiel. Deshalb eignet sie sich zur Illustration der ordonomischen Methode. Diese Methode strebt an (und leitet dazu an), das dualistische Trade-off-Denken und die damit verbundenen Diskursblockaden durch orthogonale Positionierungen zu überwinden.

[60] Viertens: Orthogonale Positionierungen fallen nicht vom Himmel. Sie müssen erarbeitet werden. Zentral hierfür ist die Erkenntnis, dass jede orthogonale Positionierung auf (mindestens) zwei Überbietungsargumenten beruht, mit denen symmetrisch beide Seiten des Meinungsspektrums erfasst werden.

[61] Fünftens: Überbietungsargumente haben eine besondere diskursive Qualität. Die besteht darin, die dem Trade-off-Denken zugrunde liegenden normativen Anliegen ernst zu nehmen, um sie in der Moraldimension ihres je eigenen Anspruchs zu übertreffen.

[62] Sechstens: Im vorliegenden Fall bedeutet das, dass sich mit dem institutionellen Reformvorschlag, die Mittelmeerroute zu schließen und als Junktim hierzu geordnete Verfahren für die Anerkennung von Asylanten, Flüchtlingen und Arbeitsmigranten einzurichten, zwei Ansprüche normativer Überbietung verbinden: (a) der Anspruch, solidarischer zu sein als eine humanitäre Hilfe, die allein auf deutschem Boden geleistet wird und damit höchst selektiv all jenen Menschen vorenthalten wird, die in Not sind, es aber nicht schaffen, ihren Fuß auf deutschen Boden zu setzen; und (b) der Anspruch, dem nationalen Interesse der Deutschen dienlicher zu sein als eine reine Abschottungspolitik, die die internationalen Interdependenzen ignoriert. Langfristig nützt es nicht, sondern schadet, wenn man sich die gedankliche Mühe sowie die beträchtliche materielle Ressourcen erfordernde Arbeit zu sparen versucht, vorausschauend und mit einer verlässlichen Orientierung an Rechtsprinzipien aktiv daran mitzuwirken, dass sich die Lebensumstände in den an die Europäische Union angrenzen Regionen, vor allem im Nahen Osten und in Afrika, nachhaltig verbessern, so dass die dort lebenden Menschen - d.h. eine extrem große Zahl von (potenziellen) Flüchtlingen - für sich und ihre Familien auf ihrem Kontinent eine rationale Bleibeperspektive entwickeln können.

[63] Siebtens: Diese konkrete Überbietungsargumentation ist - wie prinzipiell jede orthogonale Positionierung - anfällig für Missverständnisse. Gravierende Missverständnisse können insbesondere dann auftreten, wenn die jeweiligen Adressaten, auf die die ordonomische Stellungnahme diskursiv ausgerichtet ist, sich nicht darauf einlassen (wollen oder können), den Wahrnehmungsrahmen ihres wertedualistischen Trade-off-Denkens zu verlassen und eine konstruktive politische Debatte zu führen, was zwingend voraussetzt, die teilnehmenden Personen nicht in die Kategorien von Gut und Böse einzuteilen. Um es recht allgemein zu formulieren: Orthogonale Positionierungen argumentieren immer jenseits von rechts und links. Damit besteht grundsätzlich die Gefahr, zwischen die Fronten zu geraten: (a) von den Rechten als nicht-rechts und damit links sowie umgekehrt (b) von den Linken als nicht-links und damit rechts eingeordnet - und bedenkenlos 
abgelehnt - zu werden. Genau das ist ja das Vertrackte am Freund-Feind-Schema: dass Argumente, denen (intellektuell) der eigene Stallgeruch fehlt, automatisch als dem Gegner zugehörig schubladisiert werden. Die Ordonomik versucht, dieser Gefahr vorbeugend entgegenzuwirken, indem sie konzeptionell dazu anleitet, Überbietungsargumente zu generieren. Denn Überbietungsargumente machen es aufgrund ihrer besonderen diskursiven Qualität schwierig(er), dass sich der jeweilige Adressat einer Diskussion verweigert, die den Anspruch erhebt, sein je eigenes normatives Anliegen besser zu verwirklichen und in diesem Sinn mehr Moral möglich zu machen.

[64] Was ist aus dem Anwendungsbeispiel und seiner Reflexion zu lernen? Ich denke, vier Lektionen verdienen es, hier gesondert festgehalten zu werden, zumal sie auf jeweils ganz unterschiedlichen Ebenen angesiedelt sind:

[65] Erstens kann man speziell zur Flüchtlings(politik)debatte lernen, dass es für die Konsensorientierung eines politischen Diskurses fatal ist, wenn die Teilnehmer den Versuch aufgeben, sich wechselseitig zu überzeugen - wenn sie sich damit begnügen, auf eine an die eigene moralische Gemeinde gerichtete Binnenkommunikation zu setzen, die vornehmlich darin besteht, Wertebekenntnisse abzugeben, mit denen man die eigene Identität pflegt und Gruppenzugehörigkeit signalisiert. Aus ordonomischer Sicht vermisst man, dass sich die Pro-Seite an ihr Gegenüber mit Argumenten wendet, warum es im nationalen Interesse liegen könnte, noch mehr Menschenleben zu retten und aktive Krisenprävention zu betreiben. Und spiegelbildlich vermisst man, dass sich die Contra-Seite an ihr Gegenüber mit Argumenten wendet, warum es dem humanitären Hilfsanliegen entgegenkäme, das Engagement zur Rettung von Menschenleben stärker ins Ausland zu verlagern.

[66] Damit verbindet sich, zweitens, eine Lektion, die über die deutsche Flüchtlings(politik)debatte weit hinausweist, weil sie alle westlichen Demokratien und das gesamte Themenspektrum politischer Auseinandersetzungen betrifft: Es wäre extrem hilfreich, wenn nicht nur die jeweiligen Debattenteilnehmer, sondern auch das jeweilige gesellschaftliche Umfeld, in dem politische Debatten stattfinden, ein klareres Bewusstsein davon hätten, wie wichtig es ist, das intellektuelle Niveau der Auseinandersetzung nicht beliebig tief sinken zu lassen. Angesichts der neuen Medien, die oft ohne Redaktion auskommen, benötigen Demokratien eine breitgefächerte Infrastruktur, die sicherstellt, dass nicht nur einzelne Personen, sondern insbesondere auch Organisationen professionell damit beschäftigt sind, hitzige Debatten zu versachlichen und die Standards in den Diskursen hochzuhalten. Dies würde bedeuten, weitaus stärker als bisher darauf zu drängen, dass bloße Wertebekenntnisse, die darauf hinauslaufen, andere zu diskreditieren und auszugrenzen, als Argumentationsverweigerung und damit als diskursiver Regelversto $\beta$ angesehen und entsprechend mit Achtungsentzug geahndet werden. Damit ist eine zunehmend wichtige Aufgabe zivilgesellschaftlicher Selbst-Organisation markiert - von schulisch zu vermittelnder Medienkompetenz bis hin zu Vereinen und Stiftungen, die es sich zur Aufgabe machen, die formalen und informalen Rahmenbedingungen für die politische Auseinandersetzung so zu gestalten, dass eine dysfunktionale Streiteskalation nach Möglichkeit vermieden bzw. schnell korrigiert 
wird. In Hobbesschen Kategorien ausgedrückt, geht es darum, eine rhetorische Abrüstung aller Seiten zu organisieren, um einer anderenfalls drohenden (diskursiven) Anarchie zu entgehen.

[67] Die dritte Lektion betrifft eine Anforderung an die Theoriebildung. Sie besteht darin, das »Faktum des vernünftigen Pluralismus « (Rawls 1992; 2001 sowie als Überblick Pies 2016 [1994]) weitaus ernster zu nehmen, als es in manchen Teilbereichen des Wissenschaftsbetriebs üblich zu sein scheint: Es ist ein alltäglicher Normalfall, dass Streit entsteht, sobald bei politischen Themen je vernünftige, aber divergente Moralauffassungen aufeinandertreffen. Da hilft es nicht, wenn man diesen Streit in die Wissenschaft hineinträgt und dann Theorien entwickelt, die sich auf die eine oder andere Seite des Streits schlagen. So wird Wissenschaft parteiisch. Theorien identifizieren - und infizieren - sich dann mit strittigen Werturteilen, verstoßen gegen Max Webers (vgl. 1988 [1922]) Seriositätsstandard der Werturteilsfreiheit und büßen so ihren Status als Wissenschaft ein. Beträchtliche Teile der Literatur zur Ethik allgemein und insbesondere zur Wirtschafts- und Unternehmensethik könnten m.E. davon profitieren, wenn sie ihre Theoriebildung stärker darauf ausrichten würden, dieser Anforderung systematisch Rechnung zu tragen.

[68] Die vierte Lektion betrifft die Entstehungsgeschichte der Ordonomik. Sie kennzeichnet damit meinen persönlichen Umgang mit dieser Anforderung zur Theoriebildung. Ich möchte für mich in Anspruch nehmen, meine Forschungsbeiträge von Anfang an auf exakt diese Problemstellung referentialisiert zu haben: Bereits Dissertation und Habilitation bearbeiten die Kernfrage, wie die Wissenschaft angesichts des Faktums eines vernünftigen Pluralismus zu konkreten Politikproblemen konsensfördernde Stellungnahmen entwickeln kann, ohne selbst wertstrittige Werturteile zu fällen. Meine Antwort habe ich transparent ausgewiesen: als »Rationalisierung des politischen Liberalismus" bzw. als »ökonomische(n) Ansatz diskursiver Politikberatung « (so die jeweiligen Untertitel von Pies 1993; 2000a). Zugrunde liegt ein sowohl für die ökonomische als auch für die ethische Theorietradition ungewöhnlicher Umgang mit Normativität, der sich als pragmatisch und minimalistisch kennzeichnen lässt - pragmatisch, weil er empirisch vorfindliche normative Positionen aufgreift, um sie zu dekonstruieren, zu rekonstruieren und zu überbieten; minimalistisch, weil die normativen Stellungnahmen nicht apodiktisch auf normativen Setzungen beruhen, sondern auf der Hypothese, die sfor the sake of argument e eingeführt wird - und im Bedarfsfall leicht korrigiert werden kann -, dass die Adressaten die (von ihnen selbst!) in Anspruch genommene normative Position auch dann noch einsichtig finden, wenn sie mit einem Argument konfrontiert werden, das ihre Position diskursiv überbietet. 


\title{
6. Ausblick
}

\begin{abstract}
»Also muss eine in Funktionssysteme differenzierte Gesellschaft auf eine moralische Integration verzichten. Aber zugleich behält sie die kommunikative Praxis bei, Menschen durch Konditionierung von Achtung und Missachtung als ganze Personen anzusprechen. Moralische Inklusion also wie gehabt, aber ohne moralische Integration des Gesellschaftssystems. Was könnte eine Ethik dazu sagen?«
\end{abstract}

Niklas Luhmann (1990: 25)

[69] Blickt man durch die ordonomische Brille auf die Diskurse der demokratischen Öffentlichkeit (nicht nur in Deutschland), dann springen einem zahlreiche Fehl-Attributionen ins Auge: In vielen Fällen wird als Ursache gesellschaftlicher Missstände ein individuelles Motiv (`Gier der Manager $)$ ), eine organisationale Fehl-Orientierung ('Streben nach Unternehmensgewinn`) oder ein kapitalistischer System-Imperativ ( (Wettbewerb $<$ ) ausgemacht, während es vergleichsweise viel zu selten vorkommt, dass solche Missstände - wie beispielsweise Armut, Arbeitslosigkeit, Umweltverschmutzung, Korruption oder Währungs- und Finanzkrisen den situativen Anreizen einer gestaltbaren Rahmenordnung zugeschrieben werden (vgl. Pies 2015 [2009]; 2010 und 2014). Hier offenbart sich ein Mangel ökonomischer und ethischer Bildung. Die Ordonomik wurde als ein Versuch konzipiert, diesem Mangel wissenschaftlich fundiert zu begegnen. In diesem Sinne versteht sie sich als Theorie für die Praxis: als gesellschaftspolitisch relevante Analyse der Kommunikation und Implementierung moralischer Anliegen. Der Ordonomik geht es um Aufklärung und Steuerung - genauer: um Aufklärung zwecks Steuerung. Mit ihren öffentlich adressierten Argumenten für augenöffnende Perspektivwechsel und institutionelle Reformen verortet sie sich innerhalb von - z.T. blockierten - gesellschaftlichen Lernprozessen, die sie als wissenschaftliche Teilnehmerin mit Hilfe intellektueller Orientierungsangebote aktiv zu fördern versucht.

[70] In Anspielung auf das einleitend angeführte Gedankenexperiment kann man diesen Punkt vielleicht auch so verständlich machen: Für die Ordonomik ist die als Unbehagen an der Kultur verbreitete 'Weltfremdheit $<$ das Gefühl, in der Welt nicht zu Hause zu sein - ein Signum der Moderne. Insofern bearbeitet sie mit ihrer Kritik am abstrakten Sollen ein ganz bestimmtes Entfremdungs- und Entzweiungs-Phänomen (= salienation $<$ ). Als Versöhnungsstrategie setzt sie auf eine konsequente Diesseitigkeitsorientierung: Die Ordonomik versucht mit (er-)klärenden Analysen darauf hinzuwirken, dass die Bürger, die an normativen Diskursen teilnehmen, dort nicht (wider Willen: gegen ihre ureigensten Intentionen) mit außerirdisch (= salien $<$ ) anmutenden Ideal- und Reform-Vorstellungen auftreten, die sich im Praxistest konkreter Umsetzungsversuche als ‘extraterrestrisch', als welt- sowie wirklichkeitsfremd und mithin als Verschlimmbesserung erweisen - 
was leicht passieren kann, wenn man die Emotionen der ersten Natur unreflektiert als Beurteilungskriterium für die Institutionen der dritten Natur heranzieht. ${ }^{16}$

[71] Vor diesem Hintergrund besteht eine der ordonomischen Hauptbotschaften darin, auf die diskursive Qualität von Argumenten zu achten. Hier ist zu unterscheiden zwischen Bekundungsargumenten und Überbietungsargumenten. Erstere kommunizieren die eigenen Wünsche und Werturteile im Modus normativer Postulate. Letztere stellen sich auf die Adressaten der Kommunikation ein und versuchen, sie inhaltlich zu überzeugen. Am Beispiel: Bekundungsargumente fordern, dass Reiche den Lebensunterhalt der Armen mittels (möglichst hoher) Einkommenstransfers unterstützen, und zur Begründung verweisen sie auf die Bedürftigkeit der Armen. Überbietungsargumente hingegen führen für Einkommenstransfers Gründe ins Feld, warum es im Interesse der Reichen liegt, die Armen wirksam zu unterstützen. Bekundungsargumente begegnen den naheliegenden Vorbehalten der Reichen mit forcierter Normativität, d.h. mit (Fremd-)Verpflichtungsgründen, die auf ein Sollen verweisen. Überbietungsargumente hingegen begegnen ihnen mit Klugheitserwägungen, d.h. mit (Selbst-)Verpflichtungsgründen, die auf ein eigenes Wollen verweisen. Bekundungsargumente setzen auf moralische Heteronomie, Überbietungsargumente hingegen auf moralische Autonomie. Bekundungsargumente verursachen und verfestigen Diskusblockaden, während Überbietungsargumente diese überwinden.

[72] Das hier mehrfach thematisierte Phänomen eines Versagens demokratischer Politikdiskurse kommt aus ordonomischer Sicht durch moralkommunikative Entgleisungen zustande. Symptomatisch hierfür ist, dass divergierende Bekundungsargumente im Vordergrund der öffentlichen Aufmerksamkeit stehen, so dass das Denken in Trade-offs - und damit eng verbunden: das Denken in den Kategorien von Gut und Böse sowie letztlich das Denken in Freund-Feind-Schemata - überhandnimmt. Hier liegen Regelverstöße gegen die Standards rationaler Argumentation vor. Diskursblockaden bis hin zum Diskursversagen sind insofern die Folge eines intellektuell verknoteten Gesprächsfadens. Abgestimmt auf diese Diagnose empfiehlt die Ordonomik als Therapie, die Knoten wieder aufzulösen und den demokratischen Diskurs von Dissens auf Konsens umzuprogrammieren, indem man orthogonale Positionierungen erarbeitet, die auf Überbietungsargumenten basieren und damit Lernprozesse zur gesellschaftlichen Aneignung von Win-WinPotenzialen in Gang setzen bzw. in Gang halten.

[73] Für diejenigen, die sich vielleicht wundern, warum zur Illustration der ordonomischen Methode hier nicht ein i.e.S. ökonomienahes Beispiel, sondern stattdessen die Flüchtlings(politik)debatte gewählt wurde, ein kurzer Hinweis zum Schluss: Die Ordonomik versteht sich nicht nur als Wirtschafts- und Unternehmensethik im Sinne einer Angewandten Ethik, sondern sie versteht sich darüber hinaus auch als interdisziplinärer Grundlagenbeitrag: als eine methodisch reflektierte Antwort auf die radikalen Herausforderungen des zeitgenössisch dominie-

16 Zur Kennzeichnung der philosophischen Tradition von Entfremdungstheorie, an die die Ordonomik Anschluss zu halten beabsichtigt, vgl. die exzellente Darstellung von Müller (2017). 
renden Ethikparadigmas (theologischer oder philosophischer Provenienz). Dieses Mainstream-Paradigma ist dualistisch angesetzt. Es arbeitet vielfach mit einer scharfen Entgegensetzung von Interesse und Moral, von Klugheit und Moral, von Recht und Moral usw. Es verficht einen Primat des Normativen, dem oft sogar ein eigenständiger ontologischer Status zugeschrieben wird, so als gäbe es neben der Welt der Gegenstände mit all ihren Schlechtigkeiten noch eine zweite intelligible (und höherwertige) Welt der guten Gründe und des guten Willens. Radikale snaturalistische - Herausforderungen dieses Paradigmas sind jenseits der vor allem von Nicht-Philosophen wie Russell Hardin (2007) betriebenen DavidHume-Renaissance von Seiten der Sozialwissenschaften und insbesondere der Naturwissenschaften formuliert worden. Diese Herausforderungen verbinden sich mit so unterschiedlichen Namen wie Niklas Luhmann (1990), Steven Pinker (2002; 2011), Ken Binmore (2011 [2005]), Jonathan Haidt (2006; 2012), Frans de Waal (2006), Philip Kitcher (2011a; 2011b; 2014), Christopher Boehm (2012), Edward O. Wilson (2012), Paul Bloom (2013), Joshua Greene (2013), Joseph Henrich (2016) und Michael Tomasello (2016). ${ }^{17}$ Bei dieser Literatur handelt es sich um eine reichlich sprudelnde Inspirationsquelle, die die Arbeit am Forschungsprogramm der Ordonomik auch in Zukunft nachhaltig prägen wird.

\section{Literaturverzeichnis}

Aurecon (1998): Der Euro kommt zu früh, Link: http://docplayer.org/40947-Der-euro-kommtzu-frueh-9-2-1998.html (zuletzt abgerufen 16.3.2017).

Becker, G. S. (1982 [1976]): Der ökonomische Ansatz zur Erklärung menschlichen Verhaltens, in: Ders.: Der ökonomische Ansatz zur Erklärung menschlichen Verhaltens, Tübingen: Mohr Siebeck, 1-15.

Beschorner, T./Kolmar, M. (2016): Locating Ordonomics, in: Zeitschrift für Wirtschafts- und Unternehmensethik, Jg. 17/H. 3, 446-458.

Binmore, K. (2011 [2005]): Natural Justice, Oxford u.a.O.: Oxford University Press.

Bloom, P. (2013): Just Babies. The Origins of Good and Evil, New York: Broadway Books.

Boehm, C. (2012): Moral Origins. The Evolution of Virtue, Altruism, and Shame, New York: Basic Books.

Bowles, S. (2004): Microeconomics. Behavior, Institutions, and Evolution. New York u.a.O: Princeton University Press.

Boyer, P. (2011): The Fracture of an Illusion. Science and the Dissolution of Religion. Frankfurt Templeton Lectures 2008, Göttingen: Vandenhoeck \& Ruprecht.

von Ebner-Eschenbach, M. (1939 [1880]): Aphorismen. Mit einem Nachwort von Karl Krolow, Frankfurt a.M.: Insel Verlag.

17 Eine charakteristische Kurzformel für die naturalistische Herausforderung der Mainstream-Auffassung von Normativität findet sich bei Boyer (2011: 12): «Beliefs in gods and spirits are not explained by the existence of gods and spirits, but by mental assumptions about agency. Beliefs about the origin of morality are not explained by moral codes and commandments, but by the way humans' minds represent moral judgments. "In ähnlicher Weise liest man bei Gintis (2016: 2): «In behavioral ethics, we recognize that people consider moral statements to have truth values, but we consider these values as being valid only for the specific social group involved, rather than having universal scope. We thus treat ethics in a manner similar to linguistics, where grammaticality and correct usage are important and analytically tractable, yet highly specific to a particular society of speakers. « Vgl. auch Homann (2015a; 2015b). 
Fabri, A. (2000): Der schmutzige Daumen, Frankfurt a.M: Zweitausendeins.

FAZ (2004): Asylpolitik. Schily für Flüchtlingslager in Afrika, in: Frankfurter Allgemeine Zeitung vom 20.7.2004, Link: http://www.faz.net/aktuell/politik/asylpolitik-schily-fuer-fluech tlingslager-in-afrika-1178370.html (zuletzt abgerufen am 1.3.2017).

Friedman, M. (1997): The Euro: Monetary Unity To Political Disunity? Link: http://www.proje ct-syndicate.org/commentary/the-euro--monetary-unity-to-political-disunity (zuletzt abgerufen am 16.3.2017).

Gesang, B. (2017): Menschenrechte als Maßstab moralischen Wirtschaftens, Link: http://forum -wirtschaftsethik.de/menschenrechte-als-massstab-moralischen-wirtschaftens/(zuletzt abgerufen am 6.2.2017).

Gintis, H. (2009): The Bounds of Reason. Game Theory and the Unification of the Behavioral Sciences, Princeton und Oxford: Princeton University Press.

Gintis, H. (2016): Behavioral Ethics, Link: http://www.umass.edu/preferen/gintis/behavioraleth ics.pdf (zuletzt abgerufen am 8.3.2017).

Gintis, H. (2017a): Rational Choice Explained and Defended, Link: http://www.umass.edu/pre feren/gintis/rationalchoice.pdf (zuletzt abgerufen am 8.3.2017).

Gintis, H. (2017b): Individuality and Entanglement. The Moral and Material Bases of Life, Princeton, New Jersey: Princeton University Press.

Greene, J. (2013): Moral Tribes. Emotion, Reason, and the Gap between Us and Them, New York: Penguin Press.

Haidt, J. (2006): The Happiness Hypothesis. Putting Ancient Wisdom and Philosophy to the Test of Modern Science, London: Arrow Books.

Haidt, J. (2012): The Righteous Mind. Why Good People Are Divided by Politics and Religion, London u. a. O.: Penguin Books.

Hardin, R. (2007): David Hume: Moral and Political Theorist, Oxford u.a.O.: Oxford University Press.

Hartwig, K.-H./Pies, I. (1995): Rationale Drogenpolitik in der Demokratie. Wirtschaftswissenschaftliche und wirtschaftsethische Perspektiven einer Heroinvergabe, Tübingen: Mohr Siebeck.

Henrich, J. (2016): Secret of Our Success: How Culture Is Driving Human Evolution, Domesticating Our Species, and Making Us Smarter, Princeton, New Jersey: Princeton University Press.

Hobbes, T. (2014 [1642-1658]): Grundzüge der Philosophie, o.O.: Holzinger.

Hobbes, T. (1996 [1651]): Leviathan, hrsg. von J. C. A. Gaskin, Oxford: Oxford University Press.

Homann, K. (2002 [1994]): Homo oeconomicus und Dilemmastrukturen, in: Ders.: Vorteile und Anreize, hrsg. von C. Lütge, Tübingen: Mohr Siebeck, 69-93.

Homann, K. (2014): Sollen und Können. Grenzen und Bedingungen der Individualmoral, Wien: Ibera.

Homann, K. (2015a): Das Können des moralischen Sollens I. Die ökonomische Problematik, in: Ethica, Jg. 23/H. 3: 243-259.

Homann, K. (2015b): Das Können des moralischen Sollens II. Bedingungen individuellen moralischen Handelns, in: Ethica, Jg. 23/H. 4: 291-314.

Judis, J. B. (2016): The Populist Explosion: How the Great Recession Transformed American and European Politics, New York: Columbia Global Reports.

Kitcher, P. (2011a): The Ethical Project, Cambridge, Mass. and London: Harvard University Press.

Kitcher, P. (2011b): Philosophy Inside Out, in: METAPHILOSOPHY, Vol. 42/No. 3, 248260.

Kitcher, P. (2014): Is a Naturalized Ethics Possible?, in: Behaviour, Vol. 151: 245-260.

Litan, R. E. (2014): Trillion Dollar Economists. How Economists and their Ideas Have Transformed Business, Hoboken, New Jersey: John Wiley \& Sons.

Lubmann, N. (1990): Paradigm lost: Über die ethische Reflexion der Moral, Frankfurt a.M: Suhrkamp.

MacAskill, W. (2015): Doing Good Better: How Effective Altruism Can Help You Make a Difference, New York: Guardian Faber Publishing. 
McKenzie, R. B. (2010): Predictably Rational? In Search of Defenses for Rational Behavior in Economics, Berlin u.a.O: Springer.

Miller, R. L. R./Benjamin, D. K./North, D. C. (2005): The Economics of Public Issues, 14. Aufl., Boston u.a.O.: Pearson.

Müller, A. (2017): Platon und Aristoteles als Wegbereiter der praktischen Philosophie. Mit einem Ausblick auf die Aktualität der beiden Klassiker als Zeugen im hermeneutischen Verfahren zur Beglaubigung moderner Rechtsstaatlichkeit, Freiburg und München: Karl Alber.

Nietzsche, F. (1881): Morgenröte. Gedanken über die moralischen Vorurteile; 2. Buch, Aphorismus Nr. 99, S. 87, Link: http://www.nietzschesource.org/facsimiles/DFGA/M,87 (zuletzt abgerufen am 6.2.2017).

o.A. (2017): The Story of a Lucky Economist, Link: http://allegedwisdom.blogspot.de/?expref= next-blog, (zuletzt abgerufen am 3.3.2017).

Pies, I. (1993): Normative Institutionenökonomik. Zur Rationalisierung des politischen Liberalismus, Tübingen: Mohr Siebeck.

Pies, I. (2000a): Ordnungspolitik in der Demokratie. Ein ökonomischer Ansatz diskursiver Politikberatung, Tübingen: Mohr Siebeck.

Pies, I. (2000b): Wirtschaftsethik als ökonomische Theorie der Moral - Zur fundamentalen Bedeutung der Anreizanalyse für ein modernes Ethikparadigma, in: Gaertner, W. (Hrsg.): Wirtschaftsethische Perspektiven V. Methodische Ansätze, Probleme der Steuer- und Verteilungsgerechtigkeit, Ordnungsfragen, Schriften des Vereins für Socialpolitik, Band 228/V, Berlin: Duncker \& Humblot, 11-33.

Pies, I. (2009a): Moral als Heuristik. Ordonomische Schriften zur Wirtschaftsethik, Berlin: wvb.

Pies, I. (2009b): Moral als Produktionsfaktor. Ordonomische Schriften zur Unternehmensethik, Berlin: wvb.

Pies, I. (2012a): Regelkonsens statt Wertekonsens: Ordonomische Schriften zum politischen Liberalismus, Berlin: wvb.

Pies, I. (2012b): Regelkonsens statt Wertekonsens: Die Grundidee des politischen Liberalismus, in: Ders.: Regelkonsens statt Wertekonsens: Ordonomische Schriften zum politischen Liberalismus, Berlin: wvb, VII-XIV.

Pies, I. (2012c): Wie kommt die Normativität ins Spiel? - Eine ordonomische Argumentationsskizze, in: Ders.: Regelkonsens statt Wertekonsens: Ordonomische Schriften zum politischen Liberalismus, Berlin: wvb, 3-53.

Pies, I. (2013): Optimierung versus Koordinierung: Zur ordonomischen Klärung des wirtschaftsethischen Kernproblems, in: Baumbach-Knopf, C./Achatz, J./Knoepffler, N. (Hrsg.): Facetten der Ethik, (zugleich: Kritisches Jahrbuch der Philosophie, Band 15), Würzburg: Königshausen \& Neumann, 113-135.

Pies, I. (2014): Der wirtschaftsethische Imperativ lautet: Denkfehler vermeiden! - Sieben Lektionen des ordonomischen Forschungsprogramms, in: Friesen, H./Wolf, M. (Hrsg.): Ökonomische Moral oder moralische Ökonomie? Positionen zu den Grundlagen der Wirtschaftsethik, Freiburg und München: Karl Alber, 16-50.

Pies, I. (2015 [1993]): Zuwanderung und Asyl - Ein institutionenethischer Beitrag zur Klärung konzeptioneller Politikprobleme, in: Ders.: Guter Rat muss nicht teuer sein. Ordonomische Schriften zur Politikberatung, Band 2, Berlin: wvb, 143-154.

Pies, I. (2015 [2006]): Ökonomische Ethik: Zur Überwindung politischer Denk- und Handlungsblockaden, in: Ders.: Guter Rat muss nicht teuer sein. Ordonomische Schriften zur Politikberatung, Band 2, Berlin: wvb, 299-308.

Pies, I. (2015 [2009]): Wirtschaftspolitik, Soziale Sicherung und Ökonomische Ethik: Drei ordonomische Kurzartikel und zwei Grundlagenreflexionen, in: Ders.: Guter Rat muss nicht teuer sein. Ordonomische Schriften zur Politikberatung, Band 2, Berlin: wvb, 77100.

Pies, I. (2015 [2010]): Gier und Größenwahn? - Zur Wirtschaftsethik der Wirtschaftskrise, in: Ders.: Guter Rat muss nicht teuer sein. Ordonomische Schriften zur Politikberatung, Band 2, Berlin: wvb, 237-265.

Pies, I. (2015a): Guter Rat muss nicht teuer sein. Ordonomische Schriften zur Politikberatung, Berlin: wvb. 
Pies, I. (2015b): Die Ordnungsethik plädiert nicht für maßlose Gier, sondern für eine sorgsame Vermeidung intentionalistischer Fehlschlüsse, in: Zeitschrift für Wirtschafts- und Unternehmensethik, Jg. 16/H. 1, 76-80.

Pies, I. (2016 [1994]): John Rawls` politischer Liberalismus, in: ders.: Moderne Klassiker der Gesellschaftstheorie. Von Karl Marx bis Milton Friedman, Tübingen: Mohr Siebeck, 122.

Pies, I. (2016a): Moderne Klassiker der Gesellschaftstheorie. Von Karl Marx bis Milton Friedman, Tübingen: Mohr Siebeck.

Pies, I. (2016b): Individualethik versus Institutionenethik? - zur Moral (in) der Marktwirtschaft, in: Minnameier, G. (Hrsg.): Ethik und Beruf. Interdisziplinäre Zugänge, Bielefeld: wbv, 17-39.

Pies, I. (2016c): Werte-Erziehung? Wirtschafts-Unterricht? - Vier ordonomische Thesen zum schulischen Bildungsauftrag, in: Aufklärung und Kritik Jg. 16/H. 3, 34-44.

Pies, I. und Hielscher, S. (2014): Verhaltensökonomik versus Ordnungsethik? Zum moralischen Stellenwert von Dispositionen und Institutionen, in: Zeitschrift für Wirtschafts- und Unternehmensethik, Jg. 15/H. 3, 398-420.

Pinker, S. (2002): The Blank Slate. The Modern Denial of Human Nature, London u.a.O: Penguin Books.

Pinker, S. (2011): The Better Angels of Our Nature. Why Violence Has Declined, London u. a. O.: Viking Adult.

Rawls, J. (1992): Die Idee des politischen Liberalismus, Frankfurt a. M.: Suhrkamp.

Rawls, J. (2001): Justice as Fairness. A Restatement, Cambridge, Mass. und London: Harvard University Press.

Stigler, G. J./Becker, G. S. (1996 [1977]): De Gustibus Non Est Disputandum, in: Ders. Familie, Gesellschaft und Politik, hrsg. von I. Pies, Tübingen: Mohr Siebeck, 50-76.

Tomasello, M. (2016): A Natural History of Human Morality, Cambridge, Mass. und London: Harvard University Press.

de Waal, F. B. M. (2006): Primates and Philosophers. How Morality Evolved, Princeton: University Press Group Ltd.

Weber, M. (1988 [1922]): Gesammelte Aufsätze zur Wissenschaftslehre, Tübingen: UTB.

von Weizsäcker, C. C. (2016): Die Zukunft von Zuwanderung und Integration. Diskussionspapier Nr.2016-09 des Lehrstuhls für Wirtschafts- und Unternehmensethik, Halle, Link: http://wcms.itz.uni-halle.de/download.php?down=43799\&elem=3007821 (zuletzt abgerufen am 1.3.2017).

Wilson, E. O. (2012): The Social Conquest of Earth, New York and London: Norton \& Company.

Zintl, R. (1989): Der Homo Oeconomicus: Ausnahmeerscheinung in jeder Situation oder Jedermann in Ausnahmesituationen?, in: Analyse \& Kritik 11, 52-69. 